\title{
Identification of Brassica napus small RNAs responsive to infection by a necrotrophic pathogen
}

\author{
Roshan Regmi ${ }^{1,2^{*}}$, Toby E. Newman ${ }^{1}$, Lars G. Kamphuis ${ }^{1,2}$ and Mark C. Derbyshire ${ }^{1 *}$
}

\begin{abstract}
Background: Small RNAs are short non-coding RNAs that are key gene regulators controlling various biological processes in eukaryotes. Plants may regulate discrete sets of sRNAs in response to pathogen attack. Sclerotinia sclerotiorum is an economically important pathogen affecting hundreds of plant species, including the economically important oilseed B. napus. However, there are limited studies on how regulation of sRNAs occurs in the $S$. sclerotiorum and B. napus pathosystem.

Results: We identified different classes of sRNAs from B. napus using high throughput sequencing of replicated mock and infected samples at $24 \mathrm{~h}$ post-inoculation (HPI). Overall, 3999 sRNA loci were highly expressed, of which 730 were significantly upregulated during infection. These 730 up-regulated sRNAs targeted 64 genes, including disease resistance proteins and transcriptional regulators. A total of 73 conserved miRNA families were identified in our dataset. Degradome sequencing identified 2124 cleaved mRNA products from these miRNAs from combined mock and infected samples. Among these, 50 genes were specific to infection. Altogether, 20 conserved miRNAs were differentially expressed and 8 transcripts were cleaved by the differentially expressed miRNAs miR159, miR5139, and miR390, suggesting they may have a role in the S. sclerotiorum response. A miR1885-triggered disease resistance gene-derived secondary sRNA locus was also identified and verified with degradome sequencing. We also found further evidence for silencing of a plant immunity related ethylene response factor gene by a novel sRNA using 5'-RACE and RT-qPCR.
\end{abstract}

Conclusions: The findings in this study expand the framework for understanding the molecular mechanisms of the S. sclerotiorum and B. napus pathosystem at the sRNA level.

Keywords: Small RNA, micro RNA, Degradome, Fungal pathogen, B. napus, PHAS locus, TAS gene, Ta-siRNA, PhaSiRNA

\section{Background}

Small RNAs (sRNAs) are short non-coding RNAs, ranging in size from 18 to 30 nucleotides (nt), that are important for gene expression regulation and genome stability in eukaryotes [1]. There are three major sRNA

\footnotetext{
*Correspondence: roshan.regmi@postgrad.curtin.edu.au; mark.derbyshire@curtin.edu.au

${ }^{1}$ Centre for Crop and Disease Management, School of Molecular and Life Sciences, Curtin University, Bentley, WA 6102, Australia

Full list of author information is available at the end of the article
}

classes, microRNAs (miRNAs), short interfering RNAs (siRNAs) and P-element Induced WImpy (PIWI) associated RNAs (piRNAs); while the latter only occur in animals [2], the former two are found in plants. Different types of sRNAs have different biogenesis pathways [3].

sRNAs in plants silence gene expression through the RNA interference (RNAi) pathway. RNA dependent RNA polymerases (RdRps), Dicer-like proteins (DCLs), and Argonauts (AGOs) are the main RNAi pathway enzymes. RdRps facilitate the formation of dsRNAs, which

(c) The Author(s). 2021, corrected publication 2021. Open Access This article is licensed under a Creative Commons Attribution 4.0 International License, which permits use, sharing, adaptation, distribution and reproduction in any medium or format, as long as you give appropriate credit to the original author(s) and the source, provide a link to the Creative Commons licence, and indicate if changes were made. The images or other third party material in this article are included in the article's Creative Commons licence, unless indicated otherwise in a credit line to the material. If material is not included in the article's Creative Commons licence and your intended use is not permitted by statutory regulation or exceeds the permitted use, you will need to obtain permission directly from the copyright holder. To view a copy of this licence, visit http://creativecommons.org/ licenses/by/4.0/. The Creative Commons Public Domain Dedication waiver (http://creativecommons.org/publicdomain/zero/1. 0/) applies to the data made available in this article, unless otherwise stated in a credit line to the data. 
are processed into sRNAs by DCLs. In general, AGO is thought to guide one of the strands (the guide strand) of dicer-processed sRNAs to silence complementary targets [4] while the other strand (the passenger 'sRNA"' strand), is often quickly degraded. However, recent studies have shown that the passenger strand can also have important gene silencing roles in plants [5-7]. In addition to complementary pairing with transcripts, siRNAs can also regulate gene expression epigenetically by RNA-directed methylation of complementary DNA [1].

Plants have many classes of siRNA [1, 8-10]. The main siRNA classes are the hairpin-siRNAs (hp-siRNAs), natural-antisense siRNAs (natsiRNAs), secondary siRNAs and heterochromatic siRNAs (hetsiRNAs) [1]. These classes have distinct biogenesis pathways and may involve DCL protein mediated cleavage of duplexes from either very long hairpins (hp-siRNAs) or double stranded RNAs generated from single stranded precursors by RdRp enzymes [1]. In addition to silencing mRNAs, miRNA-mediated cleavage of mRNAs or noncoding RNA precursors may also produce secondary siRNAs. These are often described as phased siRNAs (phasiRNAs) as they appear at precise 21-22 nucleotide intervals from the miRNA cleavage site. Loci that produce pha-siRNAs are known as 'PHAS' loci [11]. The secondary RNAs produced by PHAS loci may silence the gene from which they are derived or they may act in trans to silence the expression of other genes; the latter type of secondary siRNAs are known as trans-acting siRNAs (ta-siRNAs) and their biogenesis loci are often referred to as 'TAS' genes.

While a large number of miRNA-triggered secondary siRNAs have been identified in the genomes of plants, only a few have been experimentally validated [12-15]. Four miRNA-triggered ta-siRNA families have been characterized in the model plant A. thaliana [13]. Of these, the miRNA390-triggered TAS3 genes were found to be conserved across various plant species. The formation of pha-siRNAs depends on several protein components, including SUPPRESSOR OF GENE SILENCING 3 (SGS3), RDR6 and DCL4 [13]. Studies on PHAS loci in different plants have shown that miRNAs trigger phasiRNA production from many types of transcript, including noncoding RNAs, and the mRNAs of disease resistance and pentratricopetide repeat genes [16]. Nucleotide-binding site leucine-rich repeat (NBS-LRR) genes form the largest set of genes identified so far that can potentially produce pha-siRNAs upon binding of specific miRNAs [17].

Small RNAs in plants regulate genes associated with various biological processes such as seed germination [18], organ development [19] and maturation [20], signal transduction [21], and stress response [22]. Plants under pathogen attack may employ various sRNA-regulated immune pathways [23, 24]. For example, while studying the sRNAome in wheat cultivars during Puccinia graminis infection, Gupta et al. (2012) reported that miR408 exhibits different expression patterns in susceptible and resistant cultivars after a two-day course of infection [25]. Some immunity-related sRNAs have also been functionally characterised. For example, in the model plant $A$. thaliana, miR393 targets different auxin signalling genes to confer antibacterial resistance [22] and miR408 is a negative regulator of plantacyanins and laccase genes [26]. These latter genes have roles in stress responses, cell-to-cell signalling and maintaining plasticity and vigour of the cell wall. In addition, overexpression of miR7695 results in an incremental increase in resistance in rice against the blast fungus Magnaporthe oryzae [27]. During viral infection of plants, changes in the accumulation of miRNAs result in production of different pha-siRNAs [28]. In legumes and tomato, a number of miRNA families are involved in triggering pha-siRNAs by binding to the transcripts of NB-LRR genes $[16,29]$. In tomato, the abundance of secondary siRNAs from disease resistance genes was lower during bacterial and viral infection, suggesting that pha-siRNA production is important for fine-tuning defence responses [29]. Recently Cui et al. (2020) demonstrated the role of miR1885-mediated ta-siRNA expression in maintaining plant growth and immunity in $B$. napus upon viral infection [30]. The roles of pha-siRNAs in plant response to bacterial and viral infection have been investigated in several studies but little is known about their roles in responding to pathogenic fungi. One of the few studies on this subject was by Wu et al. (2017). This study characterized pha-siRNAs produced by tomato in response to Botrytis cinerea infection [14]. It was found that many pathogen-responsive tomato pha-siRNAs downregulate transcription factors, which is suggestive of a broad role in the regulation of gene expression [15].

Canola (B. napus) is an economically important oilseed crop grown worldwide [31]. Sclerotinia stem rot (SSR), caused by the fungus Sclerotinia sclerotiorum, is an important disease that causes large economic losses in canola [32]. Some studies have been conducted in Brassica spp. to identify plant-specific miRNAs [33-35] under biotic and abiotic stresses. There have been two studies on B. napus miRNA expression upon S. sclerotiorum infection [36, 37]. However, these studies were performed with single sRNA libraries at 3, 12 and $48 \mathrm{~h}$ post-inoculation (HPI) without any replicates, which limits the proper understanding of differential expression of small RNAs in the B. napus response to S. sclerotiorum. Furthermore, in comparison to mature miRNAs deposited in miRBase for other plants like $M$. truncatula, O. sativa and $A$. thaliana, the number of miRNAs for $B$. napus is quite low, suggesting many miRNAs in $B$. 
napus are yet to be discovered. Finally, little is known about the triggers of PHAS loci in the B. napus genome and their functions in gene regulation in response to pathogens.

To assess differential expression of sRNAs, identify new pathogen-responsive miRNAs and characterise the role of secondary sRNAs in the B. napus response to $S$. sclerotiorum, we developed replicated sRNA libraries for mock-inoculated and S. sclerotiorum inoculated leaves $24 \mathrm{~h}$ post-inoculation to characterize different classes of sRNAs. To identify targets of these sRNAs, we also performed high throughput degradome sequencing and, for one gene, 5'RACE and RT-qPCR.

For the first time, we identified a large number of $B$. napus sRNAs up-regulated in response to $S$. sclerotiorum infection after 24 HPI. We also found evidence of pathogen-responsive activation of novel PHAS loci likely involved in regulation of disease resistance proteins. Our follow-up 5'-RACE and qPCR studies provided further evidence of sRNA-directed regulation of a gene involved in ethylene signalling.

\section{Results}

\section{Overview of sequencing results}

To determine the role of B. napus sRNAs during $S$. sclerotiorum infection we sequenced six sRNA libraries on the Illumina platform from three replicates each of mock and infected samples at 24 HPI when SSR symptoms manifested on leaves. A total of 152,090,773 raw reads were obtained from the six libraries. We retained $126,887,984$ (83.24\%) high quality reads after adapter trimming and length filtering (18-30 nt) from these six libraries (Table 1). Assignment and removal of ambiguous reads (that map to both plant and fungal genome) resulted in 41,797,278 unique $B$. napus sRNA reads that match best to the $B$. napus genome across all libraries. The reads that potentially originated from structural RNAs (rRNAs, snRNAs, snoRNAs) accounted for $\sim 5 \%$ of this total. The clean, high-quality mappable reads were then aligned to the $B$. napus genome. The overall alignment rate was $88.7 \%$ with the highest percentage mapping in the mock samples (above 98\%), while in infected samples an average of $\sim 78.9 \%$ of reads mapped, ranging from 77 to $86.3 \%$ between replicates. Among the mapped reads, $\sim 86 \%$ were mapped to more than one genomic locus revealing that these sRNAs originated from genomic repeats. From our dataset, we found 14\% of sRNA reads that uniquely mapped to a single genomic locus. Table 1 provides an overall summary of the sequencing data.

To determine the grouping of infected and mock libraries we performed a principal component analysis on mean normalized counts from DESeq2 (Fig. 1A). The principal component analysis showed the replicated datasets were well grouped for two treatment groups, i.e. mock and infected, suggesting large overall differences between these treatments. Mock and infected samples were separated along principal component 1 , which explained $99 \%$ of the variance. There was some spread between the infected samples along principal component 2. However, variance between these samples along this axis is negligible, since only $1 \%$ of the variance was explained by PC2.

\section{Characteristic features of the B. napus small RNA population}

To determine which sRNAs were induced in response to infection, we produced three sequencing replicates each from mock and infected samples. The following metrics are based on the pooled biological replicates for each treatment, mock and infected. Size class distribution and $5^{\prime}$ nucleotide bias are two important characteristics to determine the origin and activities of sRNAs. To determine whether there may be a difference in the composition of sRNA origins in mock and infected samples, we analysed the nucleotide length and 5 ' nucleotide bias of

Table 1 An overall summary of the sequencing data

\begin{tabular}{|c|c|c|c|c|c|c|c|c|c|}
\hline Sample & Raw reads & $\begin{array}{l}\text { Clean } \\
\text { filtered } \\
\text { reads }\end{array}$ & $\begin{array}{l}\text { Unique } B \text {. } \\
\text { napus } \\
\text { reads }^{\dagger}\end{array}$ & $\begin{array}{l}\text { Structural } \\
\text { RNA }^{\ddagger}\end{array}$ & $\begin{array}{l}\text { Clean } \\
\text { mappable } \\
\text { reads }^{+\dagger}\end{array}$ & $\begin{array}{l}\text { Uniquely } \\
\text { mapped } \\
\text { reads }\end{array}$ & $\begin{array}{l}\text { Reads mapping to } \\
\text { multiple locations }\end{array}$ & $\begin{array}{l}\text { Total } \\
\text { mapped to } \\
B . \text { napus }\end{array}$ & $\begin{array}{l}\text { Total mapped } \\
\text { to B. napus (\%) }\end{array}$ \\
\hline Mock & $23,754,736$ & $20,144,362$ & $8,944,885$ & 318,393 & $8,626,492$ & $1,047,207$ & $7,416,613$ & $8,463,820$ & 98.1 \\
\hline Mock & $22,377,118$ & $18,983,632$ & $7,767,860$ & 311,538 & $7,456,322$ & $1,103,293$ & $6,242,937$ & $7,346,230$ & 98.5 \\
\hline Mock & $31,509,749$ & $26,430,696$ & $11,421,524$ & 450,137 & $10,971,387$ & $1,535,228$ & $9,301,292$ & $10,836,520$ & 98.8 \\
\hline Infected & $26,483,807$ & $20,626,924$ & $3,779,014$ & 202,125 & $3,576,889$ & 445,577 & $2,310,074$ & $2,755,651$ & 77 \\
\hline Infected & $23,182,766$ & $18,818,235$ & $3,866,998$ & 223,567 & $3,643,431$ & 462,630 & $2,214,409$ & $2,677,039$ & 73.5 \\
\hline Infected & $24,782,597$ & $21,884,135$ & $6,016,997$ & 508,508 & $5,508,489$ & 570,565 & $4,180,632$ & $4,751,197$ & 86.3 \\
\hline Total & $152,090,773$ & $126,887,984$ & $41,797,278$ & $2,014,268$ & $39,783,010$ & $5,164,500$ & $31,665,957$ & $36,830,457$ & 87.3 \\
\hline
\end{tabular}

"Reads after size and adapter filtering

${ }^{\dagger}$ Reads that mapped best to the B. napus genome from Bbsplit

${ }^{\ddagger}$ Reads that potentially originated from structural RNAs (ribosomal RNA, snRNAs, e.t.c)

${ }^{++}$Reads after removing potential structural RNAs that were used for ShortStack 


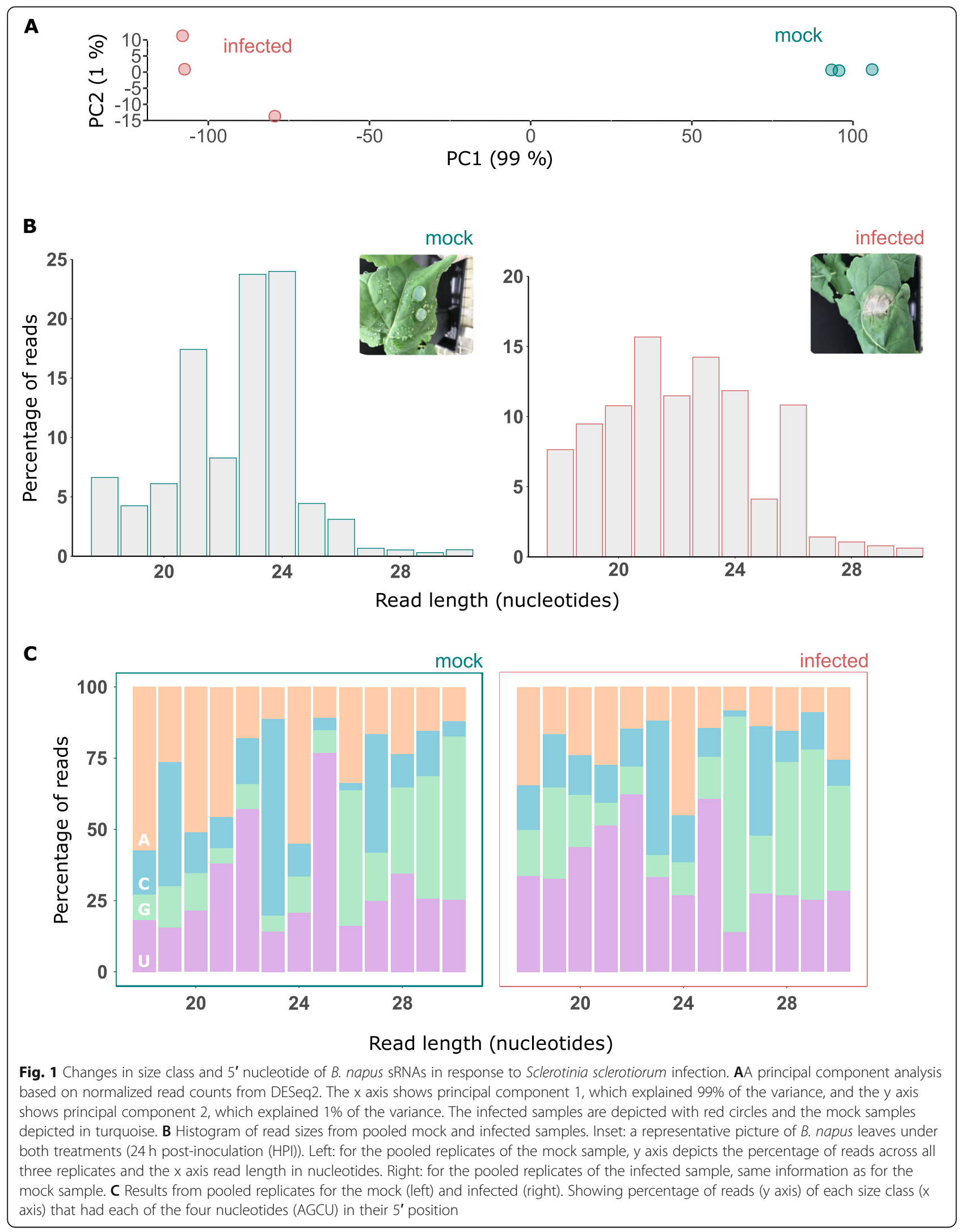


these sRNAs. Interestingly, we found a difference in length distribution between mock and infected samples (Fig. 1B), suggesting that upon infection, sRNA biogenesis mechanisms are altered. In mock samples, almost $50 \%$ of total reads belonged to 24 and 23 nucleotide (nt) sRNAs followed by $21 \mathrm{nt}$. Adenine was enriched as the $5^{\prime}$ nucleotide in $24 \mathrm{nt}$ sRNAs while cytosine was more abundant in $23 \mathrm{nt}$ sRNAs. A $5^{\prime}$ nucleotide bias toward uracil was present mostly in 22 nt sRNAs (Fig. 1C).

In infected samples, size classes were more uniform than in mock samples (Fig. 1B). The most abundant read size was $21 \mathrm{nt}$ with a slight $5^{\prime}$ uracil bias, followed by 23 nt with a slight cytosine bias (Fig. 1C). We also found a peak at $26 \mathrm{nt}$ in infected samples with a $5^{\prime}$ guanine bias. Similarly, size classes of $18,19,20$ and 22 nt were also more abundant in infected samples. However, among non-redundant reads in both samples, most were $24 \mathrm{nt}$. Apart from size distribution, the ratio of total to unique reads is also an important feature of an sRNA library [38]. The lower complexity of $21 \mathrm{nt}$ sequences in the infected samples in comparison to 24-nt sequences indicates that a small number of unique reads of $21 \mathrm{nt}$ are highly expressed while there are many different $24 \mathrm{nt}$ sequences. Such features have been attributed to $24 \mathrm{nt}$ heterochromatin sRNAs [39]. Small RNA size distributions for non-redundant reads in the mock and infected sample is shown in Supplementary Fig. 1.

Previous reports presented similar data with a $5^{\prime}$ uracil bias in $21 \mathrm{nt}$ and a $5^{\prime}$ adenine bias in $24 \mathrm{nt}$ sRNAs. The 24 nt $5^{\prime}$ adenine biased siRNAs have been previously shown to be involved in RNA dependent DNA methylation in A. thaliana with preferential loading into AGO4, while the 21 nt $5^{\prime}$ uracil biased sRNAs have preferential loading into AGO1 [40]. Overall, our results suggest a marked shift in the types of sRNAs expressed from mock to infected $B$. napus leaves.

\section{A total of 730 unique $B$. napus small RNAs are upregulated in response to Sclerotinia sclerotiorum infection}

To assess what B. napus sRNAs accumulate in response to infection with $S$. sclerotiorum, we performed a differential expression analysis with DESeq2. We did not only consider differential expression of miRNAs but the entire sRNA-ome in B. napus. ShortStack predicted 121,977 sRNA loci, 104,421 of which were likely Dicerderived. Among these loci, 3999 were highly expressed, with at least 100 raw major RNA sequencing reads (Supplementary Table 1). If these sRNAs were responding to infection, we hypothesised that they might be more expressed in infected samples as compared to mock samples. We found 915 loci significantly altered in their expression in infected samples compared to the mock samples. Among these, 730 were upregulated in $B$. napus after S. sclerotiorum infection; these loci produced 565 unique sRNAs based on the major sRNAs predicted by ShortStack (Fig. 2A).

These 730 upregulated sRNAs were mostly enriched for 20 and 21 nt sequences, while the 185 downregulated major sRNAs were enriched for $24 \mathrm{nt}$ sequences (Fig. 2B). Uracil was enriched at the $5^{\prime}$ ends of all the size classes for upregulated sRNAs except $24 \mathrm{nt}$, which had a 5' adenine bias (Fig. 2C). However, downregulated sRNAs exhibited a $5^{\prime}$ cytosine bias at $20 \mathrm{nt}$ and $21 \mathrm{nt}$ (Fig. 2C). Size classes 22 and 24 nt shared a common $5^{\prime}$ bias of uracil and adenine respectively in both sample groups. Overall, our data add weight to the hypothesis that sRNA classes with distinct biogenesis and targeting pathways were expressed in response to S. sclerotiorum challenge.

\section{Stress responsive genes are targeted by up-regulated small RNAs}

We used the degradome sequencing data to investigate targets of sRNAs upregulated during infection. A total of 64 target genes were identified from upregulated sRNAs this way (Table 2). Representative T-plots for four of these genes that were identified in infected libraries, which were assigned to different PARESnip2 confidence categories, are presented in Fig. 3. Among these 64 targets, 10 were found in both libraries, resulting in 29 and 15 unique targets for mock and infected samples, respectively. Genes that were possibly regulated by small RNAs up-regulated during infection were annotated with transcription factor-related InterPro terms such as 'ABC transcription factor', 'heat shock response', 'disease resistance protein-like', 'Zinc finger 1 domain' and 'leucine zipper domain'. This suggests that B. napus transcriptional regulatory networks may be modified by sRNAs specifically induced during infection.

\section{Identification of conserved miRNAs in B. napus}

Several miRNAs are evolutionarily conserved in the plant kingdom [41]. We assessed whether conserved $B$. napus miRNAs were expressed during S. sclerotiorum infection. Therefore, all six clean libraries were searched against miRBase (Release 22.1). From our libraries, we identified 73 conserved miRNA families with 529 mature miRNA sequences. We found that 61 miRNA families had more than one sequence while 12 miRNA families had only one mature sequence predicted (Fig. 4A). Among these miRNA families, miR156 had 42 isomiR sequences followed by miR159, and miR166 with 28 and 25 sequences, respectively. Most of these miRNA sequences were $21 \mathrm{nt}$ long, followed by 20, 19 and $18 \mathrm{nt}$ (Fig. 4B). There was a $5^{\prime}$ uracil bias in 18-22 nt long miRNA sequences, which agreed with previously reported results in miRNA studies in different plant 


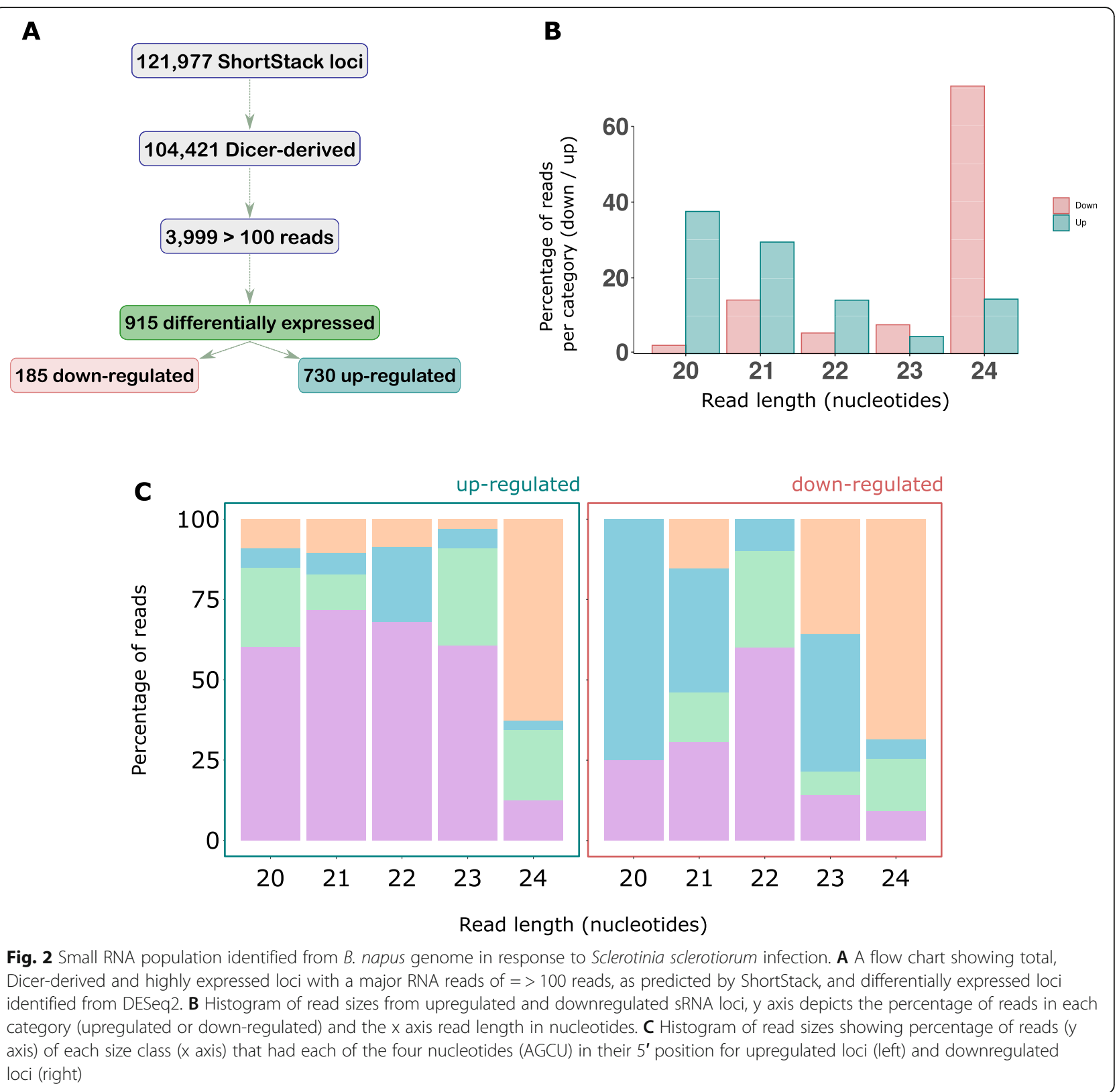

species (Fig. 4C). Using degradome sequencing, we found that from the 73 conserved miRNA families, 718 and 1406 cleaved products (Supplementary Table 2) were obtained from infected and mock libraries, respectively. Four levels of degradome cleavage site confidence, based on read mapping characteristics, are described in $[42,43]$. Category 0 is the most confident, followed by categories 1, 2 and 3. Among the 718 cleavage events in the infected sample, 507 were in category 0 , followed by category 2, 3, and 1 with 90, 87, and 34 events, respectively, based on the abundance of fragment transcripts in the library. Thus, most of the conserved miRNA targets identified with degradome sequencing were of relatively high confidence [44]. Several target genes were likely silenced by more than one miRNA family. Among these target genes, 158 non-redundant transcripts were found in infected samples. Among the 158 targets in infected samples, miR160, miR164, miR167, and miR396 were predicted to target more than 10 genes each. Similarly, miR156, miR6030, miR400, miR393, miR172 and miR171 were predicted to target eight genes (Supplementary Table 3 ). We found an additional 43 conserved miRNA families compared with $B$. napus miRNAs recorded in miRbase. These miRNAs were reported previously in several studies to regulate gene expression in plants during biotic and abiotic stress [45-48]. 
Table 2 Predicted degraded B. napus targets of upregulated B. napus small RNAs based on degradome sequencing data analysed using PARESnip2

\begin{tabular}{|c|c|c|c|c|c|c|c|c|}
\hline Gene ID & Category ${ }^{*}$ & Cleavage position & Alignment score & Duplex MFE ${ }^{\dagger}$ & Perfect MFE & MFE Ratio & $p$-value $^{\ddagger}$ & Library $^{H}$ \\
\hline BnaA08g20190D & 3 & 1211 & 3.5 & -23.6 & -32.9 & 0.717325 & 0.019978 & $M$ \\
\hline BnaA02g03600D & 0 & 748 & 4 & -20.6 & -28.9 & 0.712803 & 0.000756 & $M, I$ \\
\hline BnaC03g43110D & 2 & 219 & 4 & -24.2 & -32.3 & 0.749226 & 0.039024 & M \\
\hline BnaC05g00280D & 2 & 1144 & 3 & -24.6 & -29.6 & 0.831081 & 0.016924 & M \\
\hline BnaA10g11740D & 3 & 955 & 4 & -25.8 & -35.2 & 0.732955 & 0.032497 & M \\
\hline BnaA01g22910D & 3 & 848 & 4 & -28.4 & -39.6 & 0.717172 & 0.038079 & M \\
\hline BnaCnng67150D & 3 & 68 & 3 & -31 & -37.9 & 0.817942 & 0.01312 & M \\
\hline BnaA08g28020D & 3 & 917 & 3.5 & -29.1 & -40.8 & 0.713235 & 0.033058 & M \\
\hline BnaA07g08860D & 2 & 1153 & 1 & -32.6 & -39.2 & 0.831633 & 0.002904 & M \\
\hline BnaA09g27990D & 2 & 1156 & 1 & -32.6 & -39.2 & 0.831633 & 0.002897 & $M, I$ \\
\hline BnaC05g21250D & 2 & 1162 & 1 & -32.6 & -39.2 & 0.831633 & 0.002885 & $M, I$ \\
\hline BnaC07g11360D & 2 & 1141 & 1 & -32.6 & -39.2 & 0.831633 & 0.002929 & M \\
\hline BnaA03g36860D & 3 & 336 & 4 & -29.6 & -41.5 & 0.713253 & 0.039506 & M \\
\hline BnaA06g36560D & 1 & 345 & 2 & -33.8 & -41.5 & 0.814458 & 0.008645 & M \\
\hline BnaC07g17320D & 3 & 1383 & 2 & -33.8 & -41.5 & 0.814458 & 0.008237 & M \\
\hline BnaA04g18170D & 3 & 388 & 4 & -33.7 & -42.3 & 0.79669 & 0.025798 & M \\
\hline BnaA10g18410D & 3 & 1347 & 4 & -31.8 & -39.7 & 0.801008 & 0.021698 & M \\
\hline BnaA02g06410D & 2 & 627 & 4 & -27.5 & -39.2 & 0.701531 & 0.009812 & M \\
\hline BnaA04g07950D & 0 & 2282 & 3.5 & -31.4 & -43.5 & 0.721839 & 0.001638 & $M, I$ \\
\hline BnaA07g25390D & 0 & 2174 & 3.5 & -31.4 & -43.5 & 0.721839 & 0.001718 & $M, I$ \\
\hline BnaA08g17390D & 0 & 2282 & 4 & -30.6 & -43.5 & 0.703448 & 0.003145 & $M, I$ \\
\hline BnaA09g26170D & 0 & 2333 & 4 & -30.6 & -43.5 & 0.703448 & 0.003069 & $M, I$ \\
\hline BnaC03g59640D & 2 & 2267 & 4 & -30.6 & -43.5 & 0.703448 & 0.015732 & M \\
\hline BnaC05g23210D & 0 & 2324 & 4 & -30.6 & -43.5 & 0.703448 & 0.00308 & $M, I$ \\
\hline BnaC06g27170D & 0 & 2168 & 3.5 & -31.4 & -43.5 & 0.721839 & 0.001722 & $M, I$ \\
\hline BnaCnng25410D & 0 & 2267 & 3.5 & -31.4 & -43.5 & 0.721839 & 0.001648 & $M, I$ \\
\hline BnaC01g07210D & 3 & 37 & 4 & -33.7 & -43.2 & 0.780093 & 0.030433 & M \\
\hline BnaAnng05290D & 3 & 792 & 4 & -26.4 & -34.4 & 0.767442 & 0.009756 & M \\
\hline BnaA06g11000D & 3 & 299 & 3 & -27.3 & -33.2 & 0.822289 & 0.042121 & M \\
\hline BnaA06g11010D & 3 & 299 & 3 & -27.3 & -33.2 & 0.822289 & 0.042404 & M \\
\hline BnaC04g20940D & 3 & 215 & 0 & -33.2 & -33.2 & 1 & 0.005071 & M \\
\hline BnaC08g38450D & 1 & 1481 & 3.5 & -27.1 & -37.5 & 0.722667 & 0.001168 & M \\
\hline BnaCnng05480D & 2 & 1980 & 3.5 & -24.2 & -34.3 & 0.705539 & 0.020979 & M \\
\hline BnaC07g37000D & 2 & 487 & 0.5 & -35.5 & -35.9 & 0.988858 & 0.003279 & M \\
\hline BnaA09g16090D & 3 & 3478 & 3.5 & -28.4 & -35.2 & 0.806818 & 0.037582 & M \\
\hline BnaA04g26610D & 2 & 5713 & 4 & -23.8 & -33.9 & 0.702065 & 0.01421 & M \\
\hline BnaC04g50670D & 2 & 5713 & 4 & -23.8 & -33.9 & 0.702065 & 0.013297 & M \\
\hline BnaC01g18190D & 2 & 466 & 3 & -22.1 & -31 & 0.712903 & 0.013158 & M \\
\hline BnaCnng58300D & 3 & 891 & 4 & -26.5 & -34.4 & 0.770349 & 0.018106 & M \\
\hline BnaA02g03600D & 1 & 748 & 4 & -20.6 & -28.9 & 0.712803 & 0.003782 & $\mathrm{I}, \mathrm{M}$ \\
\hline BnaC03g33280D & 3 & 1046 & 3 & -24.3 & -28.8 & 0.84375 & 0.002259 & I \\
\hline BnaC05g38210D & 2 & 80 & 4 & -25.5 & -35.3 & 0.72238 & 0.003571 & I \\
\hline BnaA07g18970D & 1 & 840 & 3.5 & -34.6 & -46.4 & 0.74569 & 0.002773 & । \\
\hline
\end{tabular}


Table 2 Predicted degraded B. napus targets of upregulated B. napus small RNAs based on degradome sequencing data analysed using PARESnip2 (Continued)

\begin{tabular}{|c|c|c|c|c|c|c|c|c|}
\hline Gene ID & Category $^{*}$ & Cleavage position & Alignment score & Duplex MFE $^{t}$ & Perfect MFE & MFE Ratio & $p$-value ${ }^{\ddagger}$ & Library $^{H}$ \\
\hline BnaA07g26110D & 2 & 1696 & 4 & -30.9 & -39.8 & 0.776382 & 0.036546 & I \\
\hline BnaA09g27990D & 2 & 1156 & 1 & -32.6 & -39.2 & 0.831633 & 0.00145 & $\mathrm{I}, \mathrm{M}$ \\
\hline BnaC05g21250D & 2 & 1162 & 1 & -32.6 & -39.2 & 0.831633 & 0.001444 & $\mathrm{I}, \mathrm{M}$ \\
\hline BnaA07g06060D & 0 & 398 & 3.5 & -31.5 & -40.6 & 0.775862 & 0.000816 & 1 \\
\hline BnaA04g07950D & 0 & 2282 & 3.5 & -31.4 & -43.5 & 0.721839 & 0.001638 & $\mathrm{I}, \mathrm{M}$ \\
\hline BnaA07g25390D & 0 & 2174 & 3.5 & -31.4 & -43.5 & 0.721839 & 0.001718 & $\mathrm{I}, \mathrm{M}$ \\
\hline BnaA08g17390D & 0 & 2282 & 4 & -30.6 & -43.5 & 0.703448 & 0.002753 & $\mathrm{I}, \mathrm{M}$ \\
\hline BnaA09g26170D & 0 & 2333 & 4 & -30.6 & -43.5 & 0.703448 & 0.002686 & $\mathrm{I}, \mathrm{M}$ \\
\hline BnaC05g23210D & 0 & 2324 & 4 & -30.6 & -43.5 & 0.703448 & 0.002695 & $\mathrm{I}, \mathrm{M}$ \\
\hline BnaC06g27170D & 0 & 2168 & 3.5 & -31.4 & -43.5 & 0.721839 & 0.001722 & $\mathrm{I}, \mathrm{M}$ \\
\hline BnaCnng25410D & 0 & 2267 & 3.5 & -31.4 & -43.5 & 0.721839 & 0.001648 & $\mathrm{I}, \mathrm{M}$ \\
\hline BnaC03g30850D & 3 & 1850 & 3.5 & -23.1 & -29.2 & 0.791096 & 0.035487 & I \\
\hline BnaA05g25860D & 3 & 743 & 4 & -20.8 & -29.5 & 0.705085 & 0.017563 & 1 \\
\hline BnaA09g37590D & 2 & 3003 & 4 & -24.1 & -33 & 0.730303 & 0.049431 & I \\
\hline BnaA09g04340D & 3 & 45 & 4 & -25.9 & -33.3 & 0.777778 & 0.01457 & I \\
\hline BnaA08g00270D & 3 & 152 & 4 & -28 & -36 & 0.777778 & 0.021834 & I \\
\hline BnaAnng16680D & 2 & 735 & 3.5 & -34.1 & -46.4 & 0.734914 & 0.016373 & I \\
\hline BnaA06g04910D & 3 & 2188 & 4 & -24 & -30.4 & 0.789474 & 0.011989 & I \\
\hline BnaA03g18640D & 2 & 3633 & 2.5 & -25.5 & -30.4 & 0.838816 & 0.001911 & । \\
\hline BnaC08g21330D & 3 & 587 & 4 & -26.8 & -37.1 & 0.722372 & 0.013281 & I \\
\hline BnaC02g30040D & 2 & 367 & 2 & -27.8 & -34.3 & 0.810496 & 0.007331 & । \\
\hline
\end{tabular}

"Category derived from PARESnip2 based on rules of Falgren and Carrington, 2010

${ }^{t} M F E$ minimum free energy

${ }^{\ddagger} \mathrm{P}$ value based on randomisation test implemented in PARESnip2

"Library in which this tag was detected; $M$ mock library, $I$ infected library

\section{Pathogen responsive miRNAs cleave plant immune response genes in the infected sample}

To determine whether any degradation of transcripts was specific to the infected samples, we filtered out all the genes from the infected sample that were also targeted in the mock sample, resulting in 50 targets (Supplementary Table 4). Altogether, 172 Interpro domains were found in these genes. These genes had functions such as transcriptional regulation, disease resistance, and posttranscriptional gene silencing. We found several miRNAs that were shown to have a role in plant and pathogen interactions from this set also.

\section{Seventeen miRNAs belonging to 9 miRNA families were significantly upregulated during infection}

We performed differential expression analysis on the individual conserved miRNAs with raw read counts from the miRprof analysis. Among the conserved miRNAs, only 20 miRNAs were differentially expressed (Fig. 4D; Supplementary Table 5). Among these, seventeen miRNAs belonging to 9 miRNA families were upregulated during infection and three miRNAs were downregulated. Among these upregulated 9 miRNA families, miR395 had 7 isomiRs, miR169 had 3 isomiRs while miR159, miR172, miR398, miR6300, miR8155, miR8175, and miR5139 had one isomiR each. The up-regulated miRNA sequences exhibited $\log _{2}$ (fold change) values of between 1.58 and 6.13. A total of 16 of the 17 miRNAs had a $\log _{2}$ (fold change) of more than 2 .

The three downregulated miRNAs belonged to the miRNA families miR164, miR72, and miR390. These miRNAs exhibited $\log _{2}$ (fold change) values during infection of between -1.7 and - 3.59; two exhibited $\log _{2}$ (fold change) values below -2 . Interestingly, miR172 had two isomiRs with different expression patterns, with one upregulated and the another downregulated during infection.

Among the 17 upregulated miRNAs, we found 5 genes potentially cleaved by a member of the miR 159 family, miR159_27 $\left(\log _{2}(\right.$ fold change $)=1.56$; P-adjusted $\left.=0.034\right)$, and a member of the miR5139 family, miR5139_2 


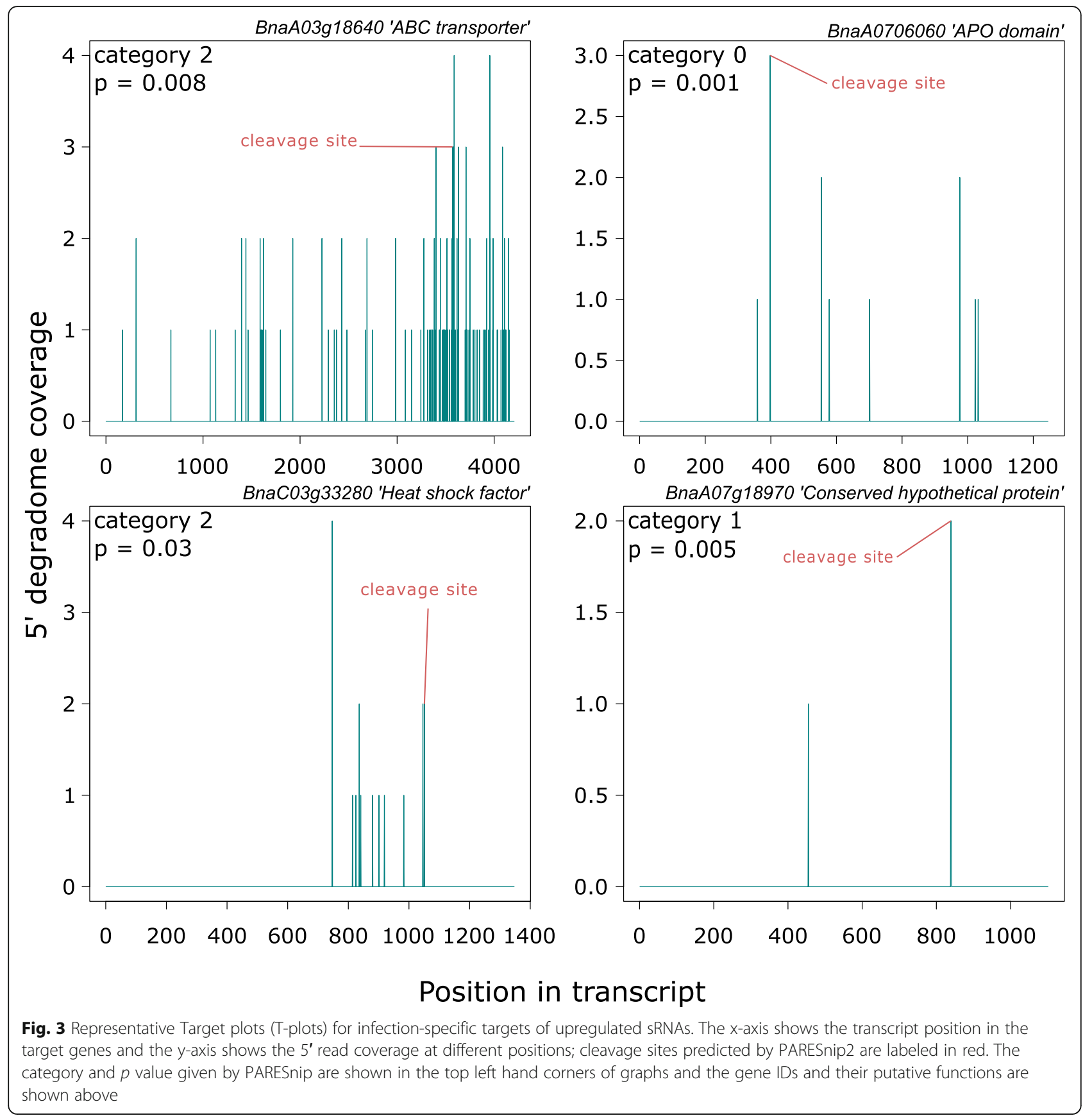

$\left(\log _{2}(\right.$ fold change $)=2.77$; P-adjusted $\left.=0.0042\right)$, in the infected samples. Surprisingly, we found 3 transcripts cleaved by downregulated miR390 member, miRNA390 $2\left(\log _{2}(\right.$ fold change $)=-2.94 ;$ P-adjusted $\left.=0.04\right)$, in the infected sample, while there was no cleavage of these genes in the mock samples.

\section{RNA structure-aided prediction algorithms identify 135} novel B. napus micro RNA loci

After filtering out the exact matches of conserved miRNAs to miRBase, 135 novel miRNA producing loci that did not have any hits in miRBase were identified from the B. napus genome. Among these miRNAs, 67 loci were found to have both passenger strand (miRNA* or 'star') and mature strand reads revealing the confidence of these novel miRNAs as per annotation criteria. A detailed description of the novel miRNA loci is given in Supplementary Table 6.

From our study, we did not find any cleavage signal from the novel miRNAs predicted from miRDeep2. We used the same set of miRNAs to predict targets using the psRNA target server. From psRNA target, 


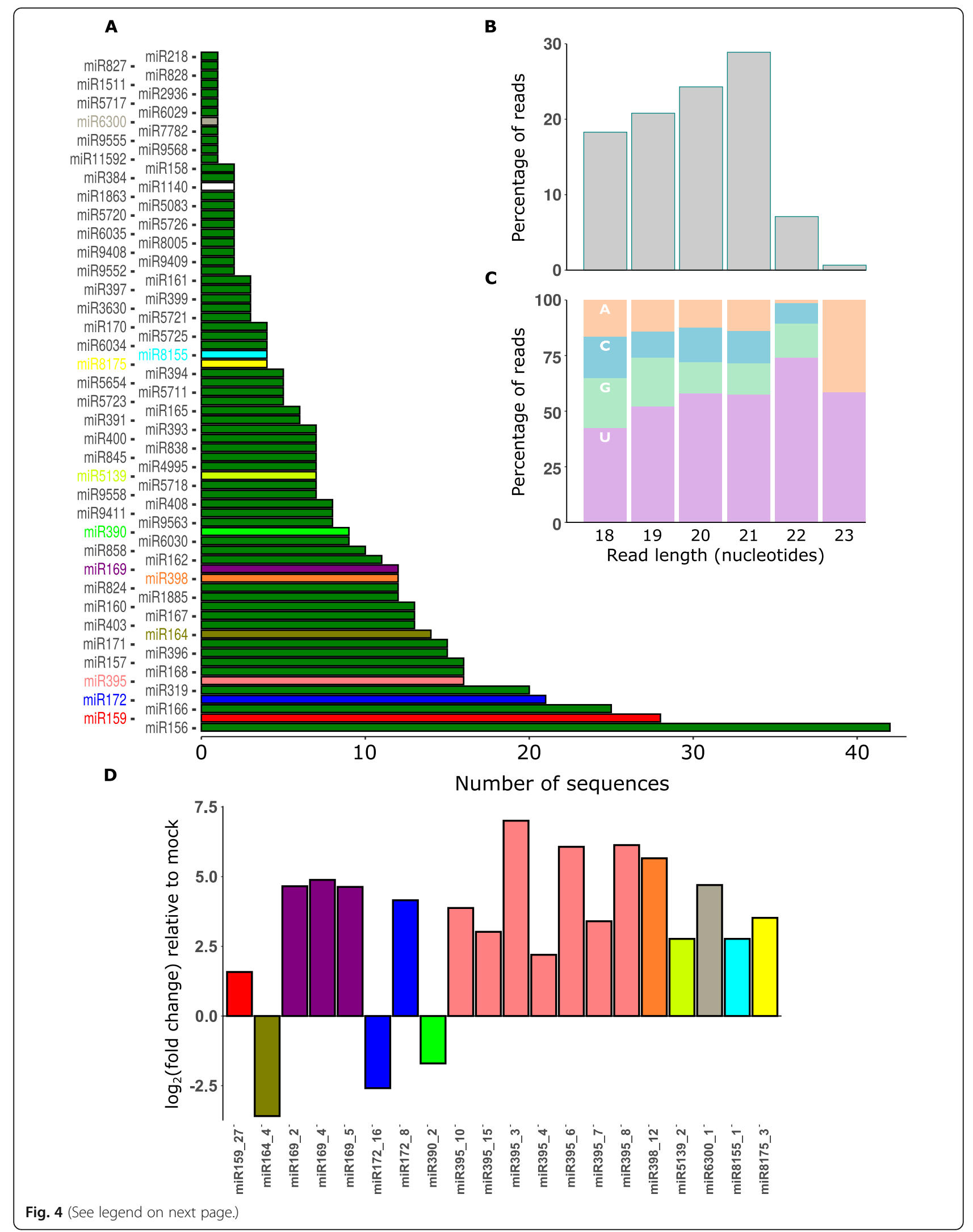


(See figure on previous page.)

Fig. 4 Prediction of infection-responsive microRNAs from the B. napus genome. A Histogram of the 73 conserved miRNA families. The $y$-axis shows the identified conserved miRNA family name and the $x$-axis shows the number of sequences (isomiRs) identified for each miRNA family; red bars are the significantly differentially expressed miRNA families. An isomiR is one of a family of highly similar miRNA sequences derived from either the guide or passenger strand. Green miRNA families were not differentially expressed. Those in other colours contained differentially expressed miRNAs and the colours correspond with D. B Histogram of read sizes from 529 conserved miRNAs. The y axis depicts the percentage of reads and the $x$ axis read length in nucleotides. C Histogram of the 529 miRNAs showing percentage of reads ( $y$ axis) of each size class ( $x$ axis) that had each of the four nucleotides (AGCU) in their $5^{\prime}$ position. D 20 differentially expressed miRNAs with $\log _{2}$ (fold change) on the $y$ axis. Colours correspond with miRNA families in $\mathbf{A}$

12,104 genes were putatively targeted by these miRNAs. Several psRNA targets might be false positives since it is entirely based on a theoretical in silico procedure, whilst a degradome signal is a better reflection of the biological cleavage. It remains to be confirmed whether these novel miRNAs have genuine targets or not.

\section{Nine B. napus PHAS loci are differentially expressed in response to Sclerotinia sclerotiorum infection}

PHAS loci have not been very well characterised in $B$. napus. Therefore, we aimed to identify expressed PHAS loci in the $B$. napus genome from our sequencing data set. We found 26 PHAS loci in the B. napus genome. The genes associated with predicted PHAS loci were annotated by aligning PHAS locus sequences to the NCBI Nucleotide Collection (nr/nt). Among the 26 PHAS genes, about half were related to disease resistance proteins (5 genes), non-coding RNAs (5 genes), and chloroplast related (3 genes). In addition, single genes were found for metal tolerance, pentatricopeptide repeat, cop9 signalosome complex subunit, and photosystem II protein D1. Nine PHAS genes were not homologous to any sequences in NCBI. A total of 182 pha-siRNAs were produced from these loci. Among these siRNAs, 41 were highly expressed, with a read abundance of more than 100 reads.

Since miRNAs are key triggers of pha-siRNA expression, we used the psRNA target server to find the cleavage sites in PHAS loci from the conserved miRNAs we identified. We found six PHAS loci potentially triggered by conserved miRNAs (Table 3). All excised PHAS clusters with their corresponding phasiRNAs are shown in Supplementary File 1. The miR390-triggered PHAS gene TAS3 was found to be conserved across different species. In this study, we found two genes possibly targeted by miR390, one of which had sequence similarity to TAS3 in $A$. thaliana.

We were able to confirm likely cleavage of one of these loci during infection using degradome sequencing (category 2, $P=0.0019$; Fig. 5A). We did not find a degradome signal for another five miRNA-triggered loci predicted with the psRNA target server. The locus we were able to validate was likely targeted by the conserved miRNA miR1885. Recently, it has been shown experimentally that miR1885 plays a key role in targeting PHAS loci residing within NBS-LRR genes to trigger ta-siRNA production [30]. Accordingly, we found that this locus had homology to NBS-LRR proteins. We identified 10 likely ta-siRNAs produced from this PHAS locus. From the degradome signal, only one of these targets BnaC05g49720D, a galactose oxidase, beta-propeller, had a cleavage signal from degradome sequencing (category $2, P=0.016$; Fig. 5B) in the infected sample. Possibly, $B$. napus miRNAs regulate gene expression in response to $S$. sclerotiorum infection through the production of miRNA triggered ta-siRNAs.

Differential expression analysis of PHAS loci showed five loci were upregulated and four were downregulated during infection. Among upregulated loci, three were related to disease resistance with a log2fold change ranging from 0.78 to 1.87 . The remaining two genes were chloroplast and photosystem II protein D1 with a log2fold change of 0.57 and 1.73, respectively. Among downregulated loci, two loci were non-coding RNAs with a $\log 2$ fold change of -0.7 and -0.96 , one was related to COP9 signalosome complex subunit (log2fold change $=$ $(-0.82)$ and the remaining one was not characterized. Figure $5 \mathrm{C}$ shows a heat map of 9 differentially expressed PHAS loci.

To gain a global overview of genes targeted by phasiRNAs we used the psRNA target server to find the targets of 41 highly expressed pha-siRNAs. We found 5918 transcripts that might be regulated by this class of sRNA. We did GO term enrichment analysis of these targets and found regulation of several biological processes (Supplementary Table 7). The terms 'posttranscriptional gene silencing' (GO:0035194), 'cellular potassium ion homeostasis' (GO:0030007), 'regulation of ARF protein signal transduction' (GO:0032012) and 'threonyl-tRNA amino acylation' (GO:0006435), 'oxidation-reduction process' (GO:0055114), and 'regulation of transcription 
Table 3 An overview of the characteristics of PHAS loci identified using PHAS tank

\begin{tabular}{|c|c|c|c|c|c|c|c|c|}
\hline PHAS locus ID & Length & $\begin{array}{l}\text { Phased } \\
\text { ratio }\end{array}$ & $\begin{array}{l}\text { Phased } \\
\text { abundance }\end{array}$ & $\begin{array}{l}\text { Phased } \\
\text { number }\end{array}$ & $\begin{array}{l}\text { Phased } \\
\text { score }\end{array}$ & $\begin{array}{l}\text { Triggering } \\
\text { miRNA }^{\mathrm{a}}\end{array}$ & Description of BLAST hit & $\begin{array}{l}\text { Differential } \\
\text { expression }\end{array}$ \\
\hline $\begin{array}{l}\text { chrCnn__ } \\
\text { random_466 }\end{array}$ & 791 & 0.398 & 3845 & 16 & 52.538 & & Uncharacterized & \\
\hline chrA04_130 & 917 & 0.448 & 274 & 19 & 47.821 & $\begin{array}{l}\text { miR4995, } \\
\text { miR6035 }\end{array}$ & chloroplast & \\
\hline chrC01_614 & 331 & 0.568 & 3417 & 7 & 32.332 & & Uncharacterized & Upregulated \\
\hline $\begin{array}{l}\text { chrA03_- } \\
\text { random_16 }\end{array}$ & 434 & 0.6 & 630 & 8 & 30.96 & & Disease resistance protein & Upregulated \\
\hline chrA01_443 & 245 & 0.79 & 877 & 5 & 26.769 & $\operatorname{miR390}$ & non coding RNA & Downregulated \\
\hline $\begin{array}{l}\text { chrUnn__ } \\
\text { random_22 }\end{array}$ & 621 & 0.468 & 159 & 11 & 26.078 & & chloroplast & \\
\hline chrC09_754 & 621 & 0.468 & 159 & 11 & 26.078 & & chloroplast & \\
\hline $\begin{array}{l}\text { chrA01__ } \\
\text { random_47 }\end{array}$ & 245 & 0.769 & 877 & 5 & 26.05 & & non coding RNA & Downregulated \\
\hline chrA07_478 & 287 & 0.621 & 823 & 6 & 25.004 & & metal tolerance protein & \\
\hline chrA01_532 & 371 & 0.666 & 111 & 7 & 21.953 & & Disease resistance protein & Upregulated \\
\hline chrC09_1169 & 392 & 0.747 & 1217 & 4 & 21.216 & $\operatorname{miR} 838$ & Uncharacterized & \\
\hline ChrA02_413 & 455 & 0.381 & 2189 & 7 & 20.533 & & Uncharacterized & \\
\hline $\begin{array}{l}\text { chrAnn_- } \\
\text { random_753 }\end{array}$ & 308 & 0.531 & 547 & 6 & 20.073 & miR390 & non coding RNA & \\
\hline chrA09_223 & 476 & 0.314 & 579 & 10 & 19.96 & miR1885 & Disease resistance protein ${ }^{b}$ & \\
\hline chrC05_811 & 392 & 0.393 & 559 & 8 & 19.894 & miR838 & non coding RNA & \\
\hline chrA01_533 & 329 & 0.746 & 288 & 4 & 16.888 & & Disease resistance protein & Upregulated \\
\hline chrC02_807 & 371 & 0.668 & 109 & 5 & 15.662 & & Disease resistance protein & \\
\hline $\begin{array}{l}\text { chrAnn_- } \\
\text { random_716 }\end{array}$ & 413 & 0.7 & 182 & 4 & 14.562 & & Uncharacterized & \\
\hline chrA10_253 & 329 & 0.376 & 198 & 7 & 13.935 & & Photosystem II protein D1 & Upregulated \\
\hline chrC01_385 & 266 & 0.444 & 237 & 5 & 12.145 & & non coding RNA & \\
\hline chrC09_1045 & 266 & 0.4 & 152 & 5 & 10.041 & & $\begin{array}{l}\text { COP9 signalosome } \\
\text { complex subunit }\end{array}$ & Downregulated \\
\hline chrC09_1044 & 434 & 0.373 & 788 & 4 & 9.949 & & Pentatricopeptide repeat & \\
\hline $\begin{array}{l}\text { chrCnn_- } \\
\text { random_695 }\end{array}$ & 391 & 0.35 & 234 & 5 & 9.558 & & Uncharacterized & \\
\hline chrA04_52 & 413 & 0.359 & 206 & 5 & 9.558 & & Uncharacterized & \\
\hline chrC08_352 & 392 & 0.46 & 118 & 4 & 8.772 & & Uncharacterized & Downregulated \\
\hline chrC07_411 & 350 & 0.305 & 175 & 4 & 6.294 & & Uncharacterized & \\
\hline
\end{tabular}

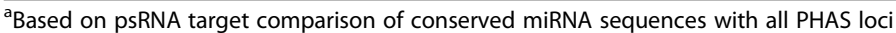

${ }^{\mathrm{b}}$ Evidence for cleavage from degradome sequencing

DNA-templated' (GO:0006355), 'carbohydrate metabolic process' (GO:0005975)' were significantly enriched.

We also specifically investigated the targets of the miR1885-triggered ta-siRNAs. We found 1601 targets of these sRNAs with psRNA target. GO term enrichment analysis showed that these ta-siRNAs possibly regulate protein phosphorylation (GO: 0006468), transcription factors (GO: 0045944), vesicle mediated transport proteins (GO:0016192), and fucose metabolic pathway genes (GO:0006004) (Supplementary Table 8).
Further analysis of sRNA targeting using 5' rapid amplification of CDNA ends and quantitative PCR

5' RACE was used to find putative cleavage sites in the novel sRNA target gene BnaA01g27570D, which is an ethylene response factor. This gene was chosen as ethylene signalling has a well-documented role in plant immunity to pathogens [42] and, although ethylene response factors have a demonstrated role in response to S. sclerotiorum [49], there is little understanding of how they might be regulated by sRNAs. This novel siRNA 


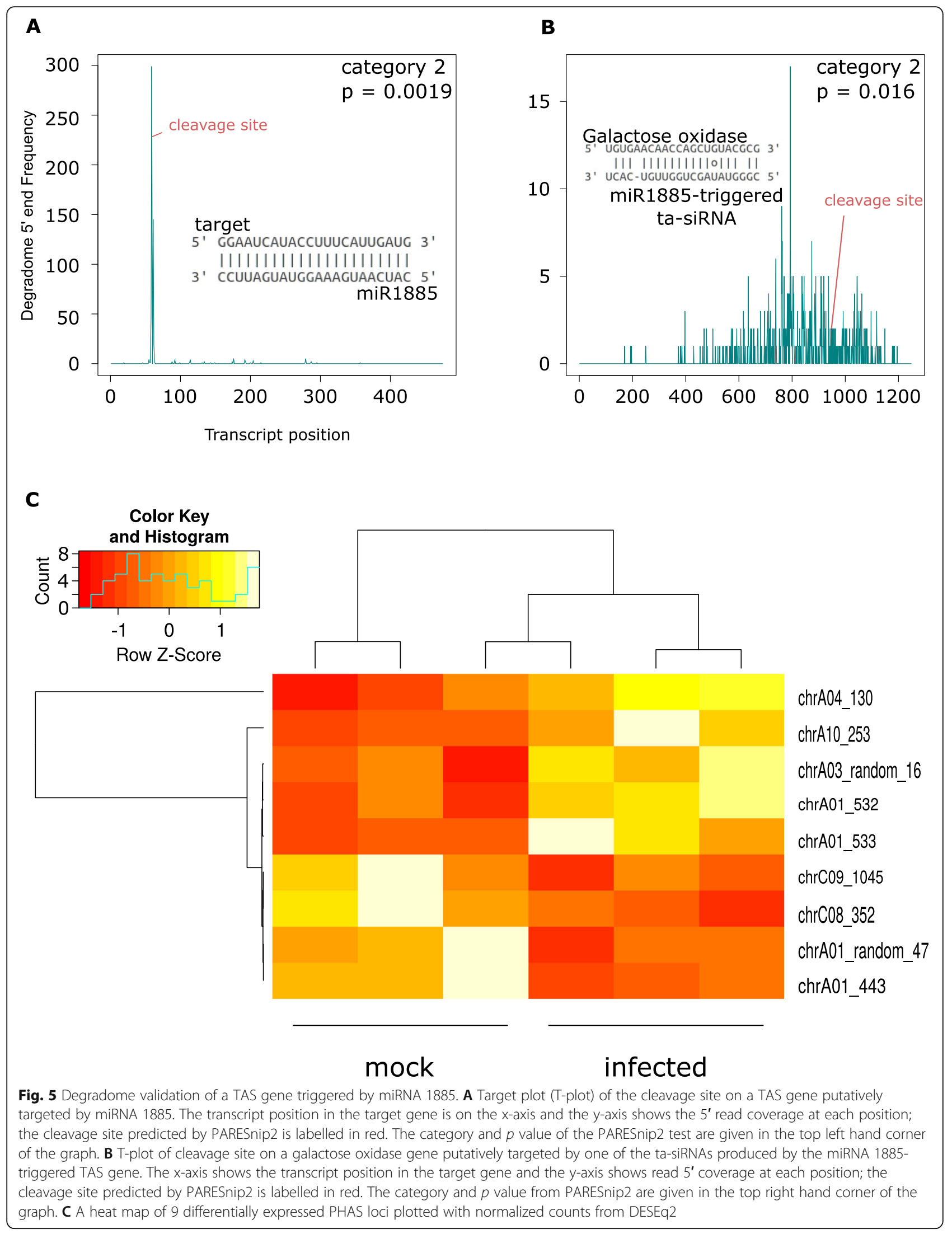




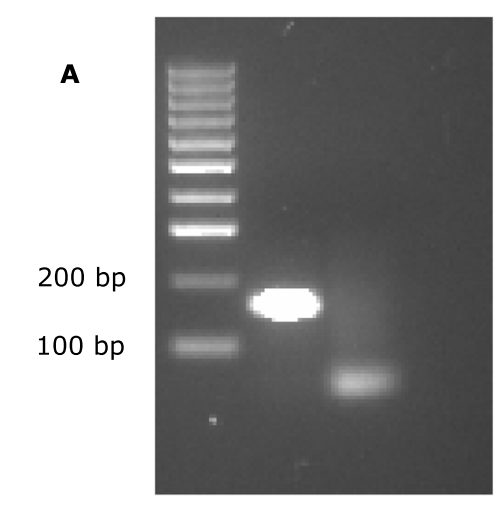

B
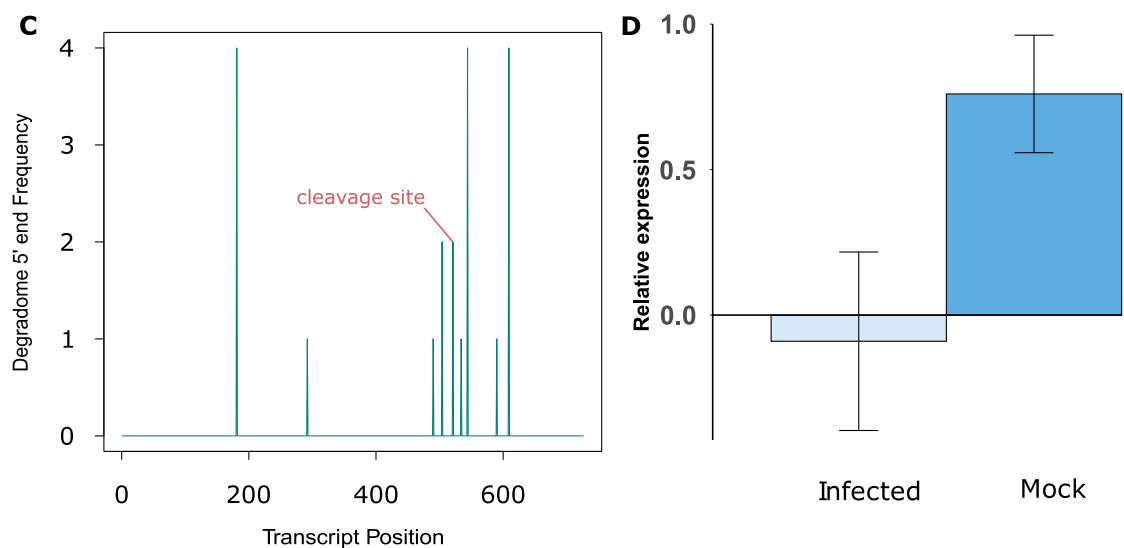

Fig. $65^{\prime}$ Rapid amplification of cDNA ends (RACE), degradome result, and qPCR for an ethylene response factor gene putatively cleaved by a novel sRNA. A Gel electrophoresis of the 5'-RACE result showing a band of the correct size; the second lane is a no template control. The full gel image is in Supplementary Fig. 1. B Sequence complementarity of the sRNA and its target. The blue arrow shows the cleavage site identified from sequencing the $5^{\prime}$-RACE product and the red arrow shows the cleavage site identified with degradome sequencing. C Target-plot (T-plot) of the degradome result of the 5'-RACE validated gene showing the transcript position in the target gene ( $x$-axis) and read 5' coverage at each position (y-axis); the cleavage site predicted by PARESnip2 is labelled in red. D RT-qPCR result of target gene in mock and infected sample ( $\mathrm{x}$-axis) and relative expression of gene to the house keeping actin gene

expressed from chromosome A01 is 22 nt long, not conserved or characterised, is neither a pha-siRNA nor a miRNA. Figure 6A shows the 5 '-RACE product of the predicted cleavage site from the infected sample; the full gel image is in Supplementary Fig. 2. A T-plot of the cleavage signal is shown in Fig. $6 \mathrm{C}$. We also assessed the expression of this target gene during infection using RT-qPCR. The average log $\left(2^{-\Delta C t}\right)$ value calculated for mock and infected sample was 0.76 and -0.09 respectively with a standard deviation of 0.35 and 0.53 Fig. 6C. The combined degradome, 5'RACE, and $\mathrm{qPCR}$ results showed that a novel $B$. napus sRNA likely regulates an ethylene response factor gene during $S$. sclerotiorum infection, leading to a decrease in its expression. Overall, the integrated degradome, 5'RACE, and RT-qPCR results showed the regulation of a plant immune response gene by a novel siRNA.

\section{Discussion}

Small RNA-omics studies have revealed the tight regulation of host immune pathways in plants [23, 50, 51]. In our study, we identified different classes of sRNAs in $B$. napus plants that responded to infection with S. sclerotiorum and showed how they may be involved in regulating different sets of genes using degradome sequencing.

We found evidence of sRNA-mediated regulation of an ethylene response factor gene (BnaA01g27570D), which we further investigated using RT-qPCR and $5^{\prime}$ RACE. The likely cleavage site identified through degradome sequencing and 5'-RACE and concomitant reduction in expression of this gene during infection suggest that it has a role in the plant response to pathogen attack. Enhanced ethylene production is an early response of plants to perception of pathogen attack, leading to 
induction of defence systems [52]. In B. napus, ethylene responsive element binding factors have been predicted to control biological processes related to defence signalling, secondary metabolite production and redox regulation. The closest homologue of BnaA01g27570D in A. thaliana, RAP2.3, has been shown to co-localise in the nucleus with another ethylene response factor, ORA59, to mediate defence to the bacterial pathogen $P$. carotovorum [53]. Since RAP2.3 has a known positive role in disease resistance and ethylene is generally found to be a positive contributor to defence, it is intriguing that our study showed a reduction in expression of BnaA01g27570D caused by potential sRNA-mediated regulation during infection. Several hypotheses could be put forward to explain this. For example, the pathogen could elicit responses in the plant that dampen ethyleneresponsive immunity. Alternatively, some components of the ethylene response system could negatively regulate immunity, as shown in some other systems [42]. Further studies are required to determine the biological significance of sRNA cleavage of this particular ethylene response factor upon S. sclerotiorum infection of B. napus.

The distribution of size classes of total sRNAs was different between mock and infected samples. The size class of sRNAs gives insights into their biogenesis, for example 21 nt sRNAs are processed by DCL1 and DCL4, whereas $22 \mathrm{nt}$ sRNAs are formed through the action of DCL2; 24 and $26 \mathrm{nt}$ sRNAs are formed by DCL3 [54]. Similarly, sRNAs with $5^{\prime}$ uracil, adenine or cytosine are loaded into AGO1, AGO2 and AGO4 or AGO5, respectively [55]. Furthermore, it has been reported previously that variation in sRNA lengths also has effects on downstream function of sRNAs [56]. In the mock sample, both redundant and non-redundant distributions peaked at $24 \mathrm{nt}$. However, in the infected sample, the redundant distribution had a major peak at $21 \mathrm{nt}$ and the nonredundant distribution had peak at $24 \mathrm{nt}$. Overall, our data suggest that a different set of sRNA biogenesis pathways is initiated upon infection with S. sclerotiorum.

A total of 730 sRNA loci were upregulated in B. napus in response to $S$. sclerotiorum infection. The upregulated sRNAs mostly belonged to the size classes of 20 and 21 nt with a 5' bias of uracil, whereas downregulated sRNAs were overrepresented for $24 \mathrm{nt}$ sequences with varied $5^{\prime}$ biases. This suggests that upon pathogen infection, the host recruits different DCL and AGO proteins for subsequent gene silencing. The upregulated sRNAs likely silenced many genes related to stress signaling, as determined from the degradome data. This suggests that the enhancement of disease symptoms might be accompanied with the negative regulation of plant immune genes during $S$. sclerotiorum infection of B. napus.

The miRNAs are sRNAs which are produced from short hairpin precursors. Based on these criteria, thousands of miRNAs have been identified and deposited in miRBase from many plants. The majority of these miRNAs show high conservation. However, only 92 mature $B$. napus miRNAs have been discovered so far. These are less numerous than those of $A$. thaliana (428), M. truncatula (756), and O. sativa (738) revealing several miRNAs are yet to be discovered in this species.

Two previous studies have assessed $S$. sclerotiorum responsive B. napus miRNAs at time points 3,12 , and 48 HPI [36, 37]. A total of 227 [36] and 77 [37] conserved miRNA sequences were identified in these studies. Both of these studies were conducted with a single sRNA sequencing library per sample. The microarray miRNA expression analysis resulted in detection of 68 infectionresponsive miRNAs, [36] and 10 of these were further analyzed with stem loop qPCR. Similarly, 10 miRNAs were found to be differentially expressed in Jian et al. [37], while only one miR166 was found to be commonly differentially expressed with respect to Cao et al. study [36]. Here, we found 529 mature conserved miRNAs belonging to 73 miRNA families, along with 20 infectioninduced miRNAs based on a replicated differential expression analysis. The enhanced number of miRNAs in our study might be due to the replicated dataset and increased sequencing depth. We did not find any common differentially expressed miRNAs in comparison to the two previous studies [36, 37]. This might be due to the different time points, $B$. napus variety, tissue collection and S. sclerotiorum strains we used. Nevertheless, the degradome data suggested these miRNAs regulate expression of transcription factors related to development and defense responses, which corroborate previous findings.

Moreover, 135 novel miRNA loci were discovered with 67 loci that had both mature and star (passenger strand) read counts. Although this adds to the overall number of B. napus miRNAs identified, we were not able to identify any likely targets of these miRNAs. This suggests that they either do not have targets or that they regulate genes through a non-cleavage mechanism such as inhibition of translation [43]..

Expression of a large number of transcription factors and auxin signaling pathway genes was likely regulated by these identified conserved miRNAs based on the infected sample degradome data. Some of these miRNAs had multiple targets in specific classes. We found 50 unique cleaved products from the infected sample that were not present in the mock samples. These genes were related to transcription factors, disease resistance proteins, and posttranscriptional silencing. For example, miR824, miR390/miR5083, miR403/miR838, miR5139, miR1885, cleaved transcripts of genes containing leucine rich repeats, zinc finger transcription factors, protein kinases, and disease resistance protein-encoding genes, 
respectively. Similarly, miR166 and miR858 cleaved transcripts of homeodomain and sant/myb domaincontaining genes, respectively. MiR824 was shown previously in the Brassicaceae to have a role in the heat stress response [57]. It is worth mentioning here miR858 has been shown to negatively regulate MYB transcription factors, thereby controlling resistance to pathogen infection in A. thaliana [58]. Similarly, plant homeodomain proteins, which are potentially regulated by miR166 in this study, control transcriptional regulation of pathogen defense-related genes [59] . These findings suggest that miRNAs are involved in regulation of multiple aspects of the immune response of B. napus to $S$. sclerotiorum.

From our study, we found 20 infection-induced miRNAs. These miRNAs were previously shown to have roles in stress responses in different pathosystems [50, 60-62]. However, we only found degradome evidence of cleavage of the transcripts of five genes, BnaA03g22590D, BnaA05g27620D, BnaCnng31260D, BnaCnng49390D, and BnaA02g31560D, which were targeted by the upregulated miRNAs miR159_27 and miR5139_2 in the infected samples. Four of these five genes were potentially cleaved by miR159_27 and the remaining gene by miR5139_2. These genes contained Interpro domains such as SANT/myb domain, homeodomain, and zinc finger domain. Surprisingly, miR390_2, whose expression was $\sim 69 \%\left(\log _{2}(0.31)=-1.7\right)$ lower in the infected sample had three target genes, whose mRNAs were cleaved only in the infected samples (BnaA06g09370D, BnaC05g49670D, BnaC08g16190D). It suggests that these genes were not expressed in the mock sample.

On the other hand, we found 11 genes whose transcripts were cleaved in the mock sample from different isomiRs of upregulated miR395, while we did not find any of these cleaved genes in the infected samples. These data suggest that gene regulation by sRNAs is quite complex and the cleavage events are not always dependent on the expression pools and other variables might also come in play.

MiR390 has been shown to have a role in the formation of ta-siRNAs and regulate auxin response factor genes [63] while miR395 plays an important role in sulphur assimilation [64]. MiR159 is present in the majority of land plants where it regulates genes encoding R2R3 MYB domain transcription factors that transduce the gibberlin (GA) signal [65] and have roles in growth, development, and biotic stress responses. It was shown recently that cotton and Arabidopsis plants accumulate increased levels of miR159 in response to the fungus Verticillium dahliae [66]. miR159 was also reported to have a role in Arabidopsis root galls under the attack of root knot nematodes, since lines lacking the miR159-
Gibberlic acid MYB pathway had better resistance to root knot nematode. Moreover, the miR159-GA MYB pathway has been shown in A. thaliana to promote the programmed cell death response [67]. Our finding of four MYB domain genes with transcripts cleaved by miR159 suggested that miR159 has a role in B. napus responses to $S$. sclerotiorum. However, further investigation will be needed to understand the precise role of this pathway.

In previous studies, miR5139 was shown to be regulated by ethylene in petal growth and was first detected in perennial herbs [68] but no specific functions were allocated to this miRNA. Later it was also shown to have tissue-specific expression in wheat [69]. However, no homolog of miR5139 was reported in B. napus to date. Here, we found the expression level of miR5139 was nearly 7 times higher $\left(\log _{2}(6.96)=2.8\right)$ during infection and it was found to cleave the transcript of the gene BnaA02g31560D which encodes a zinc finger domaincontaining gene. Zinc finger domains are reported to be present in plant resistance related proteins that are involved in effector triggered immune responses [70]. Our data shows that under the influence of $S$. sclerotiorum attack, B. napus deploys miR5139 to negatively regulate Zinc finger domain-containing genes as a defence response strategy [70].

Although we did not find any degradome cleavage signals for the other differentially expressed miRNAs, there were some interesting miRNAs identified here which were shown previously to have a role in plant responses against pathogens. miR164 was shown to manipulate programmed cell death in A. thalina. Overexpression of miR164 target genes enhanced disease symptoms [71]. miR169 was found to negatively regulate rice immunity genes during infection by the rice blast fungus [50]. miR6300 and miR8175 were highly upregulated in Alternaria-treated tomato plants compared to the control with a $\log _{2}$ (fold change) of 3.13 or 2.65. Here, we found a 3.5 and 4.7 fold increase of these two miRNAs after S. sclerotiorum infection [61].

With the aid of the degradome library we showed that miR1885 can trigger a disease resistance TAS gene which subsequently produces 10 ta-siRNAs for gene regulation. This particular miRNA was recently shown to directly silence the $B$. napus TIR-NBS-LRR resistance gene BraTNL1 and the TAS gene BraTIR1, cleavage of which generates sRNAs that regulate the photosynthesis-related gene $\mathrm{BraCP} 24$ [30]. By regulating both immunity and basal growth, this miRNA may be essential for optimizing resource allocation during development. This miRNA has been shown to be expressed at a low abundance under most conditions apart from flowering time, when the plant requires synergistic 
reductions in the levels of phytosynthesis and pathogen response. In our study, we did not observe a change in expression of miR1885 in response to $S$. sclerotiorum infection, although we observed potential cleavage of a galactose oxidase, beta propeller protein-encoding transcript (BnaC05g49720D) by one of the small RNAs derived from the TIR-NBS-LRR TAS gene it likely activates. However, such proteins have quite varied roles $[72,73]$, so it is not possible to come to any conclusions on the biological significance of this observation. Identification and elucidation of the regulatory network of pha-siRNAs is important so that the expression of these pha-siRNAs can be controlled by changing the expression of their miRNA triggers. This strategy could be useful to modulate the degree of silencing of endogenous and exogenous target genes.

\section{Conclusions}

In conclusion, our comprehensive data set allowed us to investigate overall pathogen-responsive RNA interference-based regulation of host transcripts and the actions of specific small RNA classes in response to pathogen attack. Our data suggest that targets of infection induced sRNAs including miRNAs may be associated with stress response genes. B. napus plants may differentially express both pha-siRNAs and conserved miRNAs when challenged with a necrotrophic pathogen. An integrated analysis revealed that a B. napus sRNA regulates an ethylene response factor gene during pathogen attack. Ethylene response factors regulate several jasmonate (JA) and (ET) pathways and are key players in plant innate immunity [51]. Our combined degradome, 5' RACE and qPCR results showed that the expression of one of the ethylene response genes is suppressed in $B$. napus after $S$. sclerotiorum infection. The silencing of this gene was mediated by a novel sRNA which was not characterized before.

\section{Methods}

\section{Biological materials}

An Australian S sclerotiorum isolate (CU8.24) originally collected from South Stirling WA was used for infection assays [74]. Mature sclerotia were cut into halves and placed onto $9 \mathrm{~cm}$ Petri dishes containing potato dextrose agar (PDA). After germination from the sclerotium, mycelium was subcultured onto fresh PDA medium. After $48 \mathrm{~h}$ of incubation, mycelial plugs were placed on fully expanded second or third leaves of one-month-old $B$. napus plants (AV Garnet). Seeds of AV-Garnet were originally acquired from The Australian Grains GeneBank (accession AGG95718BRAS1). The plants were grown for a month in a growth chamber with $16 \mathrm{~h}$ of daylight and $8 \mathrm{~h}$ of darkness. After infection, plants were carefully covered with a polythene bag to increase the humidity, thereby facilitating the infection process. Twenty-four HPI, a characteristic necrotic lesion was observed on the infected leaves. The infected tissues were carefully excised using sterilized scissors and immediately frozen in liquid nitrogen and stored at $-80^{\circ} \mathrm{C}$ until RNA extraction for sequencing. For mock samples, PDA only agar plugs were used without any fungal mycelium. Three leaves from three different plants were pooled together for each replicate. For small RNA sequencing, three biological replicates were sequenced separately while 2 degradome libraries were sequenced by pooling all infected replicates as one library and all mock replicates as another library.

\section{Total RNA extraction and sequencing}

Total RNAs were extracted using the TRIZOL reagent following the manufacturer's protocol (Invitrogen Carlsbad, CA, USA). After extraction, total RNAs were quantified using a Nanodrop spectrophotometer, and Qubit. The integrity of RNA samples was checked using agarose gel electrophoresis. Three to five $\mu \mathrm{g}$ and $25-30 \mu \mathrm{g}$ of total RNA were sent to Novogene (Singapore) for small RNA and degradome sequencing respectively. The sRNA sequencing was done using the NEBNext $^{\circ}$ Multiplex Small RNA Library Prep Kit for Illumina ${ }^{\circ}$ with single end $50 \mathrm{bp}$ reads according to the manufacturer's protocol. Degradome sequencing was done as mentioned in [75]. In brief, the construction of a degradome library was started from the degradation site (with monophosphate group) of the degraded mRNA. The sequencing adaptors were added to both ends of the degradation library and a library size of around $200-400 \mathrm{bp}$ was selected. The sequencing was performed on a Hiseq 2500 SE50.

\section{Analysis of small RNA sequencing data}

Raw reads were trimmed using cutadapt software (version 1.15) optimized for single-end reads with a setting of cutadapt -a (universal True Seq adapter) -m18 -M30 [76]. The quality of filtered reads was checked by running in FastQC [77]. Reads with a length in the range of $18-30 \mathrm{nt}$ were retained. Trimmed infected reads were assigned to the fungal [78] and plant [79] reference sequences using bbsplit software in the bbmap [80] program keeping ambig2 option set as toss. The option ambig2 removes all the reads that map to both references with equal confidence. The reads that were unique to the B. napus genome were kept for prediction of $B$. napus sRNAs.

For prediction of B. napus sRNA biogenesis loci, clean reads were aligned to the reference genome of $B$. napus allowing for a maximum of two mismatches. We used ShortStack [81] to gain an overall idea of sRNA producing loci from the B. napus genome and to characterize 
highly expressed sRNA loci after infection. Each library was used as a single entity without collapsing for input into ShortStack. For conserved miRNA prediction, we matched the clean reads against miRBase (version 22) (http://www.mirbase.org) using the miRProf program in UEA small RNA workbench [82]. The reads that matched to mature miRNAs in the miRbase database with 0 mismatches were considered as conserved miRNAs. The remaining reads that did not match miRBase were parsed for the prediction of novel miRNAproducing loci using the miRDeep2 program [83]. Differential expression analysis was done using the Bioconductor package DESeq2 in $\mathrm{R}$ with estimate variance - mean dependencies. We used the raw cluster abundance of 6 individual libraries from ShortStack to find differentially expressed sRNA loci. The sRNAs with a Benjamini-Hochberg corrected $p$-value of $<0.05$ were considered as differentially expressed sRNAs [84]. For the differential expression analysis of conserved miRNAs, we used the raw counts for the individual miRNA sequences identified from the miRprof program.

The phasing patterns of loci were predicted with a Perl script from the PHAS tank software (version 1.0) [85]. To find the miRNA triggered phased initiator loci, complementary cleavage sites of predicted miRNAs on PHAS loci were searched using the psRNA target server assuming that the 10th nucleotide position on the miRNA is a cleavage start position of its targeted PHAS loci [15].

\section{Analysis of sRNA targeting using in silico target prediction and degradome sequencing data}

To determine whether reads originated either from the plant or the fungus we used bbsplit [80] to categorise infected degradome reads as fungal or plant-specific reads. The filtered reads were separated from potential structural RNAs by filtering against the RFAM database [86] using the program Infernal (version 1.1.3) [87].

We used either the psRNA target server with a default setting and an expectation score of 5 for computational prediction of sRNA targets [88] or PARESnip2 [89] to validate the cleavage sites from Degradome datasets following the rules of Fahlgren and Carrington [90]. We retained the targets with category number $0-3$. Category- 0 are targets with a degraded products having degradome peaks more than one read and the maximum on the transcript where there is only one maximum. Category-1 are those having degradome peaks greater than one read and are the maximum on the transcript, but there is more than one maximum. Category- 2 peaks are those that have reads more than one and are above the average fragment abundance on the transcript. Category-3 signals are those that have greater than one read and are below or equal to the average fragment abundance on the transcript. Further verification of PHAS locus activation by specific miRNAs was also investigated using the degradome sequencing tags with PARESnip2. To gain a more detailed understanding of silencing by different sRNA classes, we used four different datasets: the one highly expressed major RNA per locus from the ShortStack program, the conserved miRNAs from miRbase, novel miRNAs annotated from miRDeep2 and phasiRNA identified by PHAS tank.

\section{Gene ontology enrichment analysis}

Gene ontology enrichment analysis was conducted on sRNA target transcripts with the topGO program from $\mathrm{R}$ 3.6.1 Bioconductor package. GO term enrichment tests were performed separately on mock and infected samples. In each case, the background set was all GO terms in the B. napus genome and the foreground set was any gene with evidence of degradome targeting. The mock and infected samples were compared to identify genes that were enriched in the mock and depleted in the infected sample or vice versa. GO terms with a $p$ value $<0.05$ were considered as significantly enriched or depleted [91].

\section{Five prime rapid amplification of CDNA ends of a cleaved target}

We conducted a 5'-RACE experiment on one of the ethylene response factor genes from our degradome dataset that is potentially cleaved by a plant sRNA. The reason for choosing this gene is directed by previous pieces of literature where these classes of genes were shown to be crucial for defence responses in plants against pathogen attack [92] and high confidence complementary site between this siRNA and the target gene according to psRNA target server. Furthermore, the sRNA targeting this gene was hitherto uncharacterised, and it is not a miRNA or phasiRNA. We used two independent samples collected from independent infection assays to conduct $5^{\prime}$ RACE using the first choice RACE kit following the manufacturer's protocol (Applied Biosystems, USA) without adding calf intestinal Phosphatase enzyme. One sample was the same as the one used for degradome sequencing while the other was not.

In brief, $5^{\prime}$ RACE adapters were ligated to $5 \mu \mathrm{g}$ of total RNA, which was reverse transcribed using the universal RT primer provided in the kit and the MMLV transcriptase. The first PCR was conducted on $1 \mu \mathrm{L}$ of cDNA with a $5^{\prime}$ outer RACE primer and gene-specific outer primer. The second nested PCR was done using the first PCR product with a $5^{\prime}$ inner RACE primer and inner nested PCR primers. The PCR product was visualized on a $2 \%$ Agarose gel. The amplified DNA fragment was gel purified and cloned into TOP TA vector and 5 independent clones were Sanger sequenced. 


\section{Quantitative polymerase chain reaction}

The expression levels of a sRNA target gene were analysed by RT-qPCR. One to five ug of total RNAs from mock and infected $B$. napus leaf samples were converted to cDNA using the MMLV reverse transcriptase kit (Sigma-Aldrich). The cDNA samples were then diluted $1 / 20$ before qPCR. The qPCR analysis was performed using the Bio-Rad Taq Universal SYBR Green Supermix according to the manufacturer's instructions. The thermocycler settings were $95^{\circ} \mathrm{C}$ for $2 \mathrm{~min}$, then $95^{\circ} \mathrm{C}$ for $15 \mathrm{~s}, 60^{\circ} \mathrm{C}$ for $30 \mathrm{~s}$ and $72^{\circ} \mathrm{C}$ for $15 \mathrm{~s}$, and cycled for 40 times, followed by $72^{\circ} \mathrm{C}$ for $2 \mathrm{~min}$. Three biological and three technical replicates were used for each sample. Relative expression was calculated as per $\log \left(2^{-\Delta C t}\right)$ method normalized to the $B$. napus housekeeping actin gene. The primers and adapters used for $5^{\prime}$ RACE and qPCR experiments are listed in Supplementary Table 9.

\author{
Abbreviations \\ ta-siRNA: Trans-acting short interfering RNA; siRNA: Short interfering RNA: \\ sRNA: Small RNA; pha-siRNA: Phased short interfering RNA; miRNA: Micro- \\ RNA; DCL: Dicer-like; natsiRNA: Natural antisense short interfering RNA; \\ hetsiRNA: Heterochromatic short interfering RNA; SSR: Sclerotinia stem rot; \\ RDR: RNA dependent RNA polymerase; RISC: RNA-induced silencing complex; \\ NBS-LRR: Nucleotide binding site leucine-rich repeat; RACE: Rapid \\ amplification of CDNA ends; qPCR: Quantitative PCR; nt: Nucleotide; \\ GO: Gene ontology
}

\section{Supplementary Information}

The online version contains supplementary material available at https://doi. org/10.1186/s12870-021-03148-6.

\section{Additional file 1: Supplementary File 1. Output of the software PHAS} Tank showing candidate PHAS loci.

Additional file 2: Supplementary Fig. 1. Size distribution of unique SRNAS.

Additional file 3: Supplementary Fig. 2. The full image of the cropped gel appearing in Fig. 6.

Additional file 4: Supplementary Table 1. All small RNA biogenesis loci identified using the program Sho:rtStack.

Additional file 5: Supplementary Table 2. Cleaved products of the 73 miRNA families. All targets identified using degradome sequencing in infected and mock samples are included.

Additional file 6: Supplementary Table 3. Targets of conserved microRNAs based on degradome sequencing data from the infected sample. Table includes genes targeted by multiple miRNAs and multiple genes targeted by the same miRNA. Targets only found in the infected sample are included.

Additional file 7: Supplementary Table 4. Genes targeted by conserved miRNAs only in the infected sample.

Additional file 8: Supplementary Table 5. Conserved micro RNA sequences with evidence of differential expression during infection.

Additional file 9: Supplementary Table 6. Known and novel miRNAs identified in this study.

Additional file 10: Supplementary Table 7. GO term enrichment analysis of 5918 transcripts possibly regulated by pha-siRNAs based on psRNA target analysis.

Additional file 11: Supplementary Table 8. GO term enrichment analysis of psRNA target-predicted targets of 1601 targets of miR1885triggered ta-siRNAs.
Additional file 12: Supplementary Table 9. Primers used in this study.

\section{Acknowledgements}

This work was supported by resources provided by the Pawsey Supercomputing Centre with funding from the Australian Government and the Government of Western Australia. Authors would like to thank Dr. Yuphin Khentry for providing technical assistance.

\section{Authors' contributions}

RR designed and analysed experiments and wrote the first manuscript draft. TEN and LGK were involved in high level and technical experimental discussions and helped develop the manuscript. MCD helped design, analyses and present experimental data, oversaw experimentation and cowrote the manuscript. All authors read and approved the final version of the manuscript.

\section{Funding}

This work was undertaken within the Centre for Crop and Disease Management (CCDM), a co-investment between Curtin University and the Grains Research and Development Commission (GRDC project number CUR00023). RR was funded by scholarships from the Australian Government Research Training Program and the Commonwealth Scientific and Industrial Organisation (CSIRO). The funders had no role in planning or conducting the research; their only role was providing funds. regulation

\section{Availability of data and materials}

The smallRNA and degradome sequencing data has been deposited in GenBank under BioProject PRJNA678586.

\section{Declarations}

Ethics approval and consent to participate

Not applicable.

\section{Consent for publication}

Not applicable.

\section{Competing interests}

The authors declare no conflict of interest.

\section{Author details}

${ }^{1}$ Centre for Crop and Disease Management, School of Molecular and Life Sciences, Curtin University, Bentley, WA 6102, Australia. ${ }^{2}$ Commonwealth Scientific and Industrial Research Organisation Agriculture and Food

Floreat WA 6014 Australia

Received: 24 November 2020 Accepted: 27 July 2021

Published online: 11 August 2021

References

1. Borges F, Martienssen RA. The expanding world of small RNAs in plants. Nat Rev Mol Cell Biol. 2015;16(12):727-41. https://doi.org/10.1038/nrm4085.

2. Ozata DM, Gainetdinov I, Zoch A, O'Carroll D, Zamore PD. PIWI-interacting RNAs: small RNAs with big functions. Nat Rev Genet. 2019;20(2):89-108. https://doi.org/10.1038/s41576-018-0073-3.

3. Guleria P, Mahajan M, Bhardwaj J, Yadav SK. Plant small RNAs: biogenesis, mode of action and their roles in abiotic stresses. Genomics Proteomics Bioinform. 201 1;9(6):183-99. https://doi.org/10.1016/S1672-0229(11)60022-3.

4. Pratt AJ, MacRae IJ. The RNA-induced silencing complex: a versatile genesilencing machine. J Biol Chem. 2009;284(27):17897-901. https://doi.org/10.1 074/jbc.R900012200.

5. Liu W-W, Meng J, Cui J, Luan Y-S. Characterization and function of MicroRNA* s in plants. Front Plant Sci. 2017;8:2200.

6. Hackenberg M, Shi B-J, Gustafson P, Langridge P. Characterization of phosphorus-regulated miR399 and miR827 and their isomirs in barley under phosphorus-sufficient and phosphorus-deficient conditions. BMC Plant Biol. 2013;13(1):1-17.

7. Trevisan S, Nonis A, Begheldo M, Manoli A, Palme K, Caporale G, et al. Expression and tissue-specific localization of nitrate-responsive miRNAs in 
roots of maize seedlings. Plant Cell Environ. 2012;35(6):1137-55. https://doi. org/10.1111/j.1365-3040.2011.02478.x.

8. Zheng Y, Wang Y, Wu J, Ding B, Fei ZJ. A dynamic evolutionary and functional landscape of plant phased small interfering RNAs. BMC Biol. 2015; 13:15

9. Xie M. Yu B: siRNA-directed DNA methylation in plants. Curr Genomics. 2015;16(1):23-31. https://doi.org/10.2174/1389202915666141128002211.

10. Howell MD, Fahlgren N, Chapman EJ, Cumbie JS, Sullivan CM, Givan SA, et al. Genome-wide analysis of the RNA-DEPENDENT RNA POLYMERASE6/ DICER-LIKE4 pathway in Arabidopsis reveals dependency on miRNA-and tasiRNA-directed targeting. Plant Cell. 2007;19(3):926-42. https://doi.org/1 0.1105/tpc.107.050062.

11. Zheng $Y$, Wang $Y$, Wu J, Ding B, Fei Z. A dynamic evolutionary and functional landscape of plant phased small interfering RNAs. BMC Biol. 2015; 13(1):32. https://doi.org/10.1186/s12915-015-0142-4.

12. de Felippes FF, Marchais A, Sarazin A, Oberlin S, Voinnet O. A single miR390 targeting event is sufficient for triggering TAS3-tasiRNA biogenesis in Arabidopsis. Nucleic Acids Res. 2017;45(9):5539-54. https://doi.org/10.1093/ nar/gkx119.

13. Felippes FF, Weigel D. Triggering the formation of tasiRNAs in Arabidopsis thaliana: the role of microRNA miR173. EMBO Rep. 2009;10(3):264-70. https://doi.org/10.1038/embor.2008.247.

14. Wu F, Chen Y, Tian X, Zhu X, Jin W. Genome-wide identification and characterization of phased small interfering RNA genes in response to Botrytis cinerea infection in Solanum lycopersicum. Sci Rep. 2017;7(1):1-10.

15. Zhang $C, L i ~ G$, Wang J, Fang J. Identification of trans-acting siRNAs and their regulatory cascades in grapevine. Bioinformatics. 2012;28(20):2561-8. https:// doi.org/10.1093/bioinformatics/bts500.

16. Zhai J, Jeong D-H, De Paoli E, Park S, Rosen BD, Li Y, et al. MicroRNAs as master regulators of the plant NB-LRR defense gene family via the production of phased, trans-acting siRNAs. Genes Dev. 2011;25(23):2540-53. https://doi.org/10.1101/gad.177527.111.

17. Deng P, Muhammad S, Cao M, Wu L. Biogenesis and regulatory hierarchy of phased small interfering RNAs in plants. Plant Biotechnol J. 2018;16(5):96575. https://doi.org/10.1111/pbi.12882.

18. Jian H, Wang J, Wang T, Wei L, Li J, Liu L. Identification of rapeseed microRNAs involved in early stage seed germination under salt and drought stresses. Front Plant Sci. 2016;7:658.

19. Achard P, Herr A, Baulcombe DC, Harberd NP. Modulation of floral development by a gibberellin-regulated microRNA. Development. 2004; 131(14):3357-65. https://doi.org/10.1242/dev.01206.

20. Guo H-S, Xie Q, Fei J-F, Chua N-H. MicroRNA directs mRNA cleavage of the transcription factor NAC1 to downregulate auxin signals for Arabidopsis lateral root development. Plant Cell. 2005;17(5):1376-86. https://doi.org/1 $0.1105 / \mathrm{tpc} .105 .030841$

21. Liu Q, Zhang Y-C, Wang C-Y, Luo Y-C, Huang Q-J, Chen S-Y, et al. Expression analysis of phytohormone-regulated microRNAs in rice, implying their regulation roles in plant hormone signaling. FEBS Lett. 2009;583(4):723-8. https://doi.org/10.1016/j.febslet.2009.01.020.

22. Navarro L, Dunoyer P, Jay F, Arnold B, Dharmasiri N, Estelle M, et al. A plant miRNA contributes to antibacterial resistance by repressing auxin signaling. Science. 2006;312(5772):436-9. https://doi.org/10.1126/science.1126088.

23. Du J, Wu G, Zhou Z, Zhang J, Li M, Sun M, et al. Identification of microRNAs regulated by tobacco curly shoot virus co-infection with its betasatellite in Nicotiana benthamiana. Virol J. 2019;16(1):30. https://virologyj.biomedcentra I.com/articles/10.1186/s12985-019-1234-5.

24. Islam W, Qasim M, Noman A, Adnan M, Tayyab M, Farooq TH, et al. Plant microRNAs: front line players against invading pathogens. Microb Pathog. 2018;118:9-17. https://doi.org/10.1016/j.micpath.2018.03.008.

25. Gupta OP, Permar V, Koundal V, Singh UD, Praveen S. MicroRNA regulated defense responses in Triticum aestivum L. during Puccinia graminis f. sp. tritici infection. Mol Biol Rep. 2012;39(2):817-24. https://doi.org/10.1007/s11 033-011-0803-5.

26. Liang $G, A i$ Q, Yu D. Uncovering miRNAs involved in crosstalk between nutrient deficiencies in Arabidopsis. Sci Rep. 2015;5(1):11813. https://doi. org/10.1038/srep11813.

27. Sánchez-Sanuy F, Peris-Peris C, Tomiyama S, Okada K, Hsing Y-I, San Segundo B, et al. Osa-miR7695 enhances transcriptional priming in defense responses against the rice blast fungus. BMC Plant Biol. 2019;19(1):1-16.

28. Peláez P, Sanchez F. Small RNAs in plant defense responses during viral and bacterial interactions: similarities and differences. Front Plant Sci. 2013;4:343.
29. Shivaprasad PV, Chen H-M, Patel K, Bond DM, Santos BA, Baulcombe DC. A microRNA superfamily regulates nucleotide binding site-leucine-rich repeats and other mRNAs. Plant Cell. 2012;24(3):859-74. https://doi.org/1 0.1105/tpc.111.095380.

30. Cui C, Wang J-J, Zhao J-H, Fang Y-Y, He X-F, Guo H-S, et al. A Brassica miRNA regulates plant growth and immunity through distinct modes of action. Mol Plant. 2020;13(2):231-45. https://doi.org/10.1016/j.molp.2019.11. 010.

31. Carré P, Pouzet A. Rapeseed market, worldwide and in Europe. OCL. 2014; 21(1):1-12.

32. Del Rio L, Bradley C, Henson R, Endres G, Hanson B, McKay K, et al. Impact of Sclerotinia stem rot on yield of canola. Plant Dis. 2007;91(2):191-4. https://doi.org/10.1094/PDIS-91-2-0191.

33. Fu Y, Mason AS, Zhang $Y$, Lin B, Xiao M, Fu D, et al. MicroRNA-mRNA expression profiles and their potential role in cadmium stress response in Brassica napus. BMC Plant Biol. 2019;19(1):1-20.

34. Wei W, Li G, Jiang X, Wang Y, Ma Z, Niu Z, et al. Small RNA and degradome profiling involved in seed development and oil synthesis of Brassica napus. PLoS One. 2018;13(10):e0204998. https://doi.org/10.1371/journal.pone.0204 998.

35. Shen D, Suhrkamp I, Wang Y, Liu S, Menkhaus J, Verreet JA, et al. Identification and characterization of microRNAs in oilseed rape (Brassica napus) responsive to infection with the pathogenic fungus Verticillium longisporum using Brassica AA (Brassica rapa) and CC (Brassica oleracea) as reference genomes. New Phytol. 2014;204(3):577-94. https://doi.org/1 0.1111/nph.12934.

36. Cao JY, Xu YP, Zhao L, Li SS, Cai XZ. Tight regulation of the interaction between Brassica napus and Sclerotinia sclerotiorum at the microRNA level. Plant Mol Biol. 2016;92(1-2):39-55. https://doi.org/10.1007/s11103-016-04 94-3.

37. Jian H, Ma J, Wei L, Liu P, Zhang A, Yang B, et al. Integrated mRNA, sRNA, and degradome sequencing reveal oilseed rape complex responses to Sclerotinia sclerotiorum (lib.) infection. Sci Rep. 2018;8(1):1-17.

38. Omidvar V, Mohorianu I, Dalmay T, Fellner M. Identification of miRNAs with potential roles in regulation of anther development and male-sterility in 7B1 male-sterile tomato mutant. BMC Genomics. 2015;16(1):1-16.

39. Schwach F, Moxon S, Moulton V, Dalmay T. Deciphering the diversity of small RNAs in plants: the long and short of it. Brief Funct Genomics Proteomics. 2009;8(6):472-81. https://doi.org/10.1093/bfgp/elp024.

40. Ma X, Wiedmer J, Palma-Guerrero J. Small RNA bidirectional crosstalk during the interaction between wheat and Zymoseptoria tritici. Front Plant Sci. 2020;10:1669. https://doi.org/10.3389/fpls.2019.01669.

41. Luo X, Gao Z, Shi T, Cheng Z, Zhang Z, Ni Z. Identification of miRNAs and their target genes in peach (Prunus persica L.) using high-throughput sequencing and degradome analysis. PLoS One. 2013;8(11):e79090.

42. Pieterse CM, Van der Does D, Zamioudis C, Leon-Reyes A, Van Wees SC. Hormonal modulation of plant immunity. Annu Rev Cell Dev Biol. 2012; 28(1):489-521. https://doi.org/10.1146/annurev-cellbio-092910-154055.

43. Li S, Liu L, Zhuang X, Yu Y, Liu X, Cui X, et al. MicroRNAs inhibit the translation of target mRNAs on the endoplasmic reticulum in Arabidopsis. Cell. 2013;153(3):562-74. https://doi.org/10.1016/j.cell.2013.04.005.

44. Addo-Quaye C, Miller W, Axtell MJ. CleaveLand: a pipeline for using degradome data to find cleaved small RNA targets. Bioinformatics. 2008; 25(1):130-1. https://doi.org/10.1093/bioinformatics/btn604.

45. Cui J, Sun Z, Li J, Cheng D, Luo C, Dai C. Characterization of miRNA160/164 and their targets expression of beet (Beta vulgaris) seedlings under the salt tolerance. Plant Mol Biol Report. 2018;36(5-6):790-9. https://doi.org/10.1007/ s11105-018-1118-7.

46. Wang Y, Li K, Chen L, Zou Y, Liu H, Tian Y, et al. MicroRNA167-directed regulation of the auxin response factors GmARF8a and GmARF8b is required for soybean nodulation and lateral root development. Plant Physiol. 2015;168(3):984-99. https://doi.org/10.1104/pp.15.00265.

47. Lu Y, Feng Z, Liu X, Bian L, Xie H, Zhang C, et al. MiR393 and miR390 synergistically regulate lateral root growth in rice under different conditions. BMC Plant Biol. 2018;18(1):261. https://doi.org/10.1186/s12870-018-1488-x.

48. Wu G, Park MY, Conway SR, Wang J-W, Weigel D, Poethig RS. The sequential action of miR156 and miR172 regulates developmental timing in Arabidopsis. Cell. 2009;138(4):750-9. https://doi.org/10.1016/j.cell.2009.06.031.

49. Girard IJ, Tong C, Becker MG, Mao X, Huang J, de Kievit T, et al. RNA sequencing of Brassica napus reveals cellular redox control of Sclerotinia infection. J Exp Bot. 2017;68(18):5079-91. https://doi.org/10.1093/jxb/erx338. 
50. Li Y, Zhao S-L, Li J-L, Hu X-H, Wang H, Cao X-L, et al. Osa-miR169 negatively regulates rice immunity against the blast fungus Magnaporthe oryzae. Front Plant Sci. 2017;8:2.

51. Soto-Suárez M, Baldrich P, Weigel D, Rubio-Somoza I, San Segundo B. The Arabidopsis miR396 mediates pathogen-associated molecular patterntriggered immune responses against fungal pathogens. Sci Rep. 2017;7(1): 44898. https://doi.org/10.1038/srep44898.

52. van Loon LC, Geraats BP, Linthorst HJ. Ethylene as a modulator of disease resistance in plants. Trends Plant Sci. 2006;11(4):184-91. https://doi.org/10.1 016/j.tplants.2006.02.005

53. Kim NY, Jang YJ, Park OK. AP2/ERF family transcription factors ORA59 and RAP2. 3 interact in the nucleus and function together in ethylene responses. Front Plant Sci. 2018;9:1675.

54. Ossowski S, Schwab R, Weigel D. Gene silencing in plants using artificial microRNAs and other small RNAs. Plant J. 2008;53(4):674-90. https://doi. org/10.1111/j.1365-313X.2007.03328.x.

55. Kim VN. Sorting out small RNAs. Cell. 2008;133(1):25-6. https://doi.org/10.1 016/j.cell.2008.03.015.

56. Wu L, Zhou H, Zhang Q, Zhang J, Ni F, Liu C, et al. DNA methylation mediated by a microRNA pathway. Mol Cell. 2010;38(3):465-75. https://doi. org/10.1016/j.molcel.2010.03.008.

57. Szaker HM, Darkó É, Medzihradszky A, Janda T. Liu H-c, Charng Y-y, Csorba T: miR824/AGAMOUS-LIKE16 module integrates recurring environmental heat stress changes to fine-tune Poststress development. Front Plant Sci. 2019;10:1454. https://doi.org/10.3389/fpls.2019.01454.

58. Camargo-Ramírez R, Val-Torregrosa B, San Segundo B. MiR858-mediated regulation of flavonoid-specific MYB transcription factor genes controls resistance to pathogen infection in Arabidopsis. Plant Cell Physiol. 2018; 59(1):190-204. https://doi.org/10.1093/pcp/pcx175.

59. Korfhage U, Trezzini GF, Meier I, Hahlbrock K, Somssich IE. Plant homeodomain protein involved in transcriptional regulation of a pathogen defense-related gene. Plant Cell. 1994;6(5):695-708. https://doi.org/10.1105/ tpc.6.5.695.

60. Zhang Q, Li Y, Zhang Y, Wu C, Wang S, Hao L, et al. Md-miR156ab and mdmiR395 target WRKY transcription factors to influence apple resistance to leaf spot disease. Front Plant Sci. 2017:8:526.

61. Sarkar D, Maji RK, Dey S, Sarkar A, Ghosh Z, Kundu P. Integrated miRNA and mRNA expression profiling reveals the response regulators of a susceptible tomato cultivar to early blight disease. DNA Res. 2017;24(3):235-50. https:// doi.org/10.1093/dnares/dsx003.

62. Gao F, Wang N, Li H, Liu J, Fu C, Xiao Z, et al. Identification of droughtresponsive microRNAs and their targets in Ammopiptanthus mongolicus by using high-throughput sequencing. Sci Rep. 2016;6(1):1-16.

63. Xia R, Xu J, Meyers BC. The emergence, evolution, and diversification of the miR390-TAS3-ARF pathway in land plants. Plant Cell. 2017;29(6):1232-47. https://doi.org/10.1105/tpc.17.00185.

64. Matthewman CA, Kawashima CG, Húska D, Csorba T, Dalmay T. Kopriva S: miR395 is a general component of the sulfate assimilation regulatory network in Arabidopsis. FEBS Lett. 2012;586(19):3242-8. https://doi.org/10.1 016/j.febslet.2012.06.044.

65. Millar AA, Lohe A, Wong G: Biology and function of miR159 in plants. Plants 2019, 8(8):255

66. Zhang T, Zhao Y-L, Zhao J-H, Wang S, Jin Y, Chen Z-Q, et al. Cotton plants export microRNAs to inhibit virulence gene expression in a fungal pathogen. Nat Plants. 2016;2(10):1-6.

67. Alonso-Peral MM, Li J, Li Y, Allen RS, Schnippenkoetter W, Ohms S, White RG, Millar AA: The MicroRNA159-Regulated GAMYB-like Genes Inhibit Growth and Promote Programmed Cell Death. Plant Physiol. 2010;154(2): 757-71. https://doi.org/10.1104/pp.110.160630. Epub 2010 Aug 10.

68. Yang Y, Chen X, Chen J, Xu H, Li J, Zhang Z: Differential miRNA expression in Rehmannia glutinosa plants subjected to continuous cropping. BMC Plant Biol 2011, 11(1):1-11, 53, DOI: https://doi.org/10.1186/1471-2229-11-53.

69. Pandey R, Joshi G, Bhardwaj AR, Agarwal M, Katiyar-Agarwal S. A comprehensive genome-wide study on tissue-specific and abiotic stressspecific miRNAs in Triticum aestivum. PLoS One. 2014;9(4):e95800. https:// doi.org/10.1371/journal.pone.0095800.

70. Cabot C, Martos S, Llugany M, Gallego B, Tolrà R, Poschenrieder C. A role for zinc in plant defense against pathogens and herbivores. Front Plant Sci. 2019;10:1171. https://doi.org/10.3389/fpls.2019.01171.

71. Lee MH, Jeon HS, Kim HG, Park OK. An Arabidopsis NAC transcription factor NAC4 promotes pathogen-induced cell death under negative regulation by
microRNA164. New Phytol. 2017;214(1):343-60. https://doi.org/10.1111/ nph.14371.

72. Chen CK-M, Chan N-L, Wang AH-J. The many blades of the $\beta$-propeller proteins: conserved but versatile. Trends Biochem Sci. 2011;36(10):553-61. https://doi.org/10.1016/j.tibs.2011.07.004.

73. Šola K, Gilchrist EJ, Ropartz D, Wang L, Feussner I, Mansfield SD, et al. RUBY, a putative galactose oxidase, influences pectin properties and promotes cell-to-cell adhesion in the seed coat epidermis of Arabidopsis. Plant Cell. 2019:31(4):809-31. https://doi.org/10.1105/tpc.18.00954.

74. Denton-Giles M, Derbyshire MC, Khentry Y, Buchwaldt L, Kamphuis LG. Partial stem resistance in Brassica napus to highly aggressive and genetically diverse Sclerotinia sclerotiorum isolates from Australia. Can J Plant Pathol. 2018:1-11.

75. Ma Z, Coruh C, Axtell MJ. Arabidopsis lyrata small RNAs: transient MIRNA and small interfering RNA loci within the Arabidopsis genus. Plant Cell. 2010;22(4):1090-103. https://doi.org/10.1105/tpc.110.073882.

76. Martin M. Cutadapt removes adapter sequences from high-throughput sequencing reads. EMBnet journal. 2011;17(1):10-2. https://doi.org/10.14806/ ej.17.1.200.

77. Andrews S: FastQC: a quality control tool for high throughput sequence data. In.: Babraham Bioinformatics, Babraham Institute, Cambridge, United Kingdom; 2010

78. Derbyshire M, Denton-Giles M, Hegedus D, Seifbarghy S, Rollins J, van Kan J, et al. The complete genome sequence of the Phytopathogenic fungus Sclerotinia sclerotiorum reveals insights into the genome architecture of broad host range pathogens. Genome Biol Evol. 2017:9(3):593-618. https:// doi.org/10.1093/gbe/evx030.

79. Chalhoub B. Early allopolyploid evolution in the post-Neolithic Brassica napus oilseed genome (vol 348, 1260782, 2014). Science. 2014;345(6202): 1255 .

80. Bushnell BBAF, Accurate, Splice-Aware Aligner. United States. : BBMap: A Fast, Accurate, Splice-Aware Aligner. United States. . 2014.

81. Axtell MJ. ShortStack: comprehensive annotation and quantification of small RNA genes. Rna. 2013;19(6):740-51. https://doi.org/10.1261/rna.035279.112.

82. Mohorianu I, Stocks MB, Applegate CS, Folkes L, Moulton V. The UEA smal RNA workbench: a suite of computational tools for small RNA analysis. In: MicroRNA Detection and Target Identification: Humana Press, NY; 2017. p. 193-224.

83. Friedländer MR, Mackowiak SD, Li N, Chen W. Rajewsky N: miRDeep2 accurately identifies known and hundreds of novel microRNA genes in seven animal clades. Nucleic Acids Res. 2012;40(1):37-52. https://doi.org/10.1 093/nar/gkr688.

84. Love MI, Huber W, Anders S. Moderated estimation of fold change and dispersion for RNA-seq data with DESeq2. Genome Biol. 2014;15(12):550. https://doi.org/10.1186/s13059-014-0550-8.

85. Guo Q, Qu X, Jin W. PhaseTank: genome-wide computational identification of phasiRNAs and their regulatory cascades. Bioinformatics. 2015;31(2):2846. https://doi.org/10.1093/bioinformatics/btu628.

86. Kalvari I, Nawrocki EP, Argasinska J, Quinones-Olvera N, Finn RD, Bateman A, et al. Non-coding RNA analysis using the Rfam database. Curr Protoc Bioinformatics. 2018;62(1):e51. https://doi.org/10.1002/cpbi.51.

87. Nawrocki EP, Eddy SR. Infernal 1.1: 100-fold faster RNA homology searches. Bioinformatics. 2013;29(22):2933-5. https://doi.org/10.1093/bioinformatics/ btt509.

88. Dai X, Zhao PX. psRNATarget: a plant small RNA target analysis server. Nucleic Acids Res, suppl_2, W159. 2011;39:W155.

89. Thody J, Folkes L, Medina-Calzada Z, Xu P, Dalmay T, Moulton V. PAREsnip2: a tool for high-throughput prediction of small RNA targets from degradome sequencing data using configurable targeting rules. Nucleic Acids Res. 2018;46(17):8730-9. https://doi.org/10.1093/nar/gky609.

90. Fahlgren N, Carrington JC. miRNA target prediction in plants. In: Plant MicroRNAs: Springer; 2010. p. 51-7.

91. Alexa A: Rahnenfuhrer, J. topGO: enrichment analysis for gene ontology ( $R$ package version 2.40. 0). Bioconductor; 2020.

92. Huang P-Y, Catinot J, Zimmerli L. Ethylene response factors in Arabidopsis immunity. J Exp Bot. 2016;67(5):1231-41. https://doi.org/10.1093/jxb/erv518.

\section{Publisher's Note}

Springer Nature remains neutral with regard to jurisdictional claims in published maps and institutional affiliations. 